\title{
Chloroquine reduces osteoclastogenesis in murine osteoporosis by preventing TRAF3 degradation
}

\author{
Yan Xiu, ${ }^{1}$ Hao Xu, ${ }^{1}$ Chen Zhao, ${ }^{1}$ Jinbo Li, ${ }^{1}$ Yoshikazu Morita, ${ }^{1}$ Zhenqiang Yao, ${ }^{1,2}$ \\ Lianping Xing, ${ }^{1,2}$ and Brendan F. Boyce ${ }^{1,2}$ \\ ${ }^{1}$ Department of Pathology and Laboratory Medicine, and ${ }^{2}$ Center for Musculoskeletal Research, \\ University of Rochester Medical Center, Rochester, New York, USA.
}

\begin{abstract}
The cytokines RANKL and TNF activate NF- $\mathrm{KB}$ signaling in osteoclast precursors (OCPs) to induce osteoclast $(O C)$ formation. Conversely, TNF can limit OC formation through NF- $\kappa B$ p100, which acts as an inhibitor, and TNF receptor-associated receptor 3 (TRAF3); however, a role for TRAF3 in RANKL-mediated OC formation is unknown. We found that TRAF3 limits RANKL-induced osteoclastogenesis by suppressing canonical and noncanonical NF- $\mathrm{\kappa B}$ signaling. Conditional OC-specific Traf3-KO (cKO) mice had mild osteoporosis and increased OC formation. RANKL induced TRAF3 degradation via the lysosome/autophagy system. The autophagy/lysosome inhibitor chloroquine reduced RANKL-induced OC formation and function by increasing TRAF3 expression in OCPs in vitro and in vivo. Although chloroquine had no effect on basal bone resorption, it inhibited parathyroid hormone- and ovariectomy-induced OC activation in WT, but not cKO, mice. Deletion of the transcription factor gene Relb resulted in increased TRAF3 expression in OCPs, which was associated with decreased RANKL-induced TRAF3 degradation. RelB directly increased expression of BECN1, a key autophagy regulator, by binding to its promoter. These data indicate that autophagic/lysosomal degradation of TRAF3 is an important step in RANKL-induced NF- $\kappa B$ activation in OCPs. Furthermore, treatments that increase TRAF3 levels in OCPs, including pharmacological inhibition of its degradation with compounds such as chloroquine, may limit bone destruction in common bone diseases.
\end{abstract}

\section{Introduction}

Bone homeostasis is maintained by a delicate balance between osteoclastic bone resorption and osteoblastic bone formation. Disruption of this balance with a higher rate of bone resorption occurs in metabolic and inflammatory bone disorders, such as postmenopausal osteoporosis and RA, resulting in increased risk of fracture and joint destruction, respectively (1). Osteoclasts (OCs) resorb bone in physiologic and pathologic conditions (2), and their formation and activity are increased in osteoporosis and RA as a result of increased production of proinflammatory cytokines, such as TNF and RANKL, which activate NF-кB (3).

$\mathrm{NF}-\kappa \mathrm{B}$ transcriptional factors regulate immune and numerous other cellular responses and play critical roles in skeletal development, endochondral ossification, $\mathrm{OC}$ and osteoblast functions, and common bone diseases (4). In mammals, there are $5 \mathrm{NF}-\kappa \mathrm{B}$ family members: RelA (p65), RelB, and c-Rel, and the precursor proteins NF-кB1 (p105) and NF-кB2 (p100), which are processed into p50 and p52, respectively (5). Canonical NF- $\kappa B$ signals through IKK $\beta$ and NF- $\mathrm{BB}$ essential modulator (NEMO), leading to phosphorylation of I $\mathrm{B} \alpha$ and translocation of $\mathrm{p} 65 / \mathrm{p} 50$ heterodimers into nuclei in response to cytokines; noncanonical NF-KB stimulates IKK $\alpha$-mediated phosphorylation of $\mathrm{p} 100$ through activation of NF- $\mathrm{KB}-$ inducing kinase (NIK) and leads to processing of p100 to p52 and release of RelB/p52 complexes. Noncanonical signaling is induced by TNF family members, including RANKL, CD40 ligand, BAFF, and lymphotoxin- $\beta$ (5).

A role for NF- $\mathrm{KB}$ signaling in bone was first identified in reports of defective cytokine-induced osteoclastogenesis in NF-кB1/2

Conflict of interest: The authors have declared that no conflict of interest exists. Citation for this article: J Clin Invest. 2014;124(1):297-310. doi:10.1172/JCI66947.
double-KO mice $(6,7)$, but NF-кB-induced osteoclastogenesis by cytokines remains incompletely understood. NF- $\mathrm{\kappa B}$ is a pivotal regulator of RANKL- and TNF-induced osteoclast precursor (OCP) differentiation and is activated via TNF receptor-associated factors (TRAFs), which share a common C-terminal structural TRAF domain that mediates oligomerization and receptor binding. RANKL activates both canonical and noncanonical NF-кB signaling in OCPs through TRAF6 and NIK, leading to activation of $\operatorname{IKK} \beta$ and $\operatorname{IKK} \alpha$, respectively $(4,8)$, and to stimulatory and inhibitory signaling in the cells (5). Similarly, TNF stimulates OC formation directly, but it also limits RANKL-and TNF-mediated OC formation by increasing cellular levels of NF- $\kappa$ B p100 (9) and other inhibitory proteins $(4,10)$. TNF typically induces fewer OCs than RANKL in vitro when WT OCPs are used, but it induces similar numbers of OCs from $N f k b 2^{-/-}$OCPs as RANKL and also robust OC formation in $\mathrm{Rankl}^{-/-}$and $\mathrm{Rank}^{-/-}$mice deficient in p100. These findings indicate that p100 inhibits TNF-induced osteoclastogenesis by a TNF receptor-associated receptor 3-dependent (TRAF3-dependent) mechanism (9), but the mechanism whereby TRAF3 inhibits osteoclastogenesis has been unclear, although it does involve NIK degradation (11).

Apart from their role as adaptor proteins, TRAFs also act as E3 ubiquitin ligases, a function crucial for the activation of NF- $\mathrm{KB}$ signaling (12). Overexpression and genetic studies identified positive activation roles for TRAF2, -5 , and -6 in canonical NF- $\kappa B$ signaling (13-15). However, study of the role of TRAF3 in NF- $\kappa$ B signaling has been difficult because most Traf3-KO mice die within the first week after birth (16). A report that TRAF3 is constitutively bound to NIK and mediates NIK ubiquitination and degradation in B cells identified a critical negative regulatory role of TRAF3 
in noncanonical signaling (17). Furthermore, $\operatorname{Traf3}^{-/-}$mice have marked B cell accumulation of NIK, and compound deficiency of NF-кB p100 rescued their early postnatal lethality (18). Although deficiency of NIK or its downstream signaling molecule, RelB, appears to have little effect on basal bone homeostasis, $\mathrm{Nik}^{-/-}$ and $\mathrm{RelB}^{-/-}$mice have defective osteoclastogenesis in response to pathologic osteolysis in vivo $(8,19)$. Additionally, transgenic mice with OC-targeted overexpression of a mutant NIK lacking the TRAF3-binding domain have decreased bone mass and increased bone erosion in a serum-transfer model of RA (11). Collectively, these data indicate that NIK and TRAF3 play important negative regulatory roles in noncanonical NF- $\mathrm{KB}$ signaling.

\section{Results}

Mice with OC-specific deletion of TRAF3 have increased osteoclastogenesis and osteoporosis, mediated by increased canonical and noncanonical $N F-\kappa B$ signaling. Culture of WT BM cells with M-CSF for 2 days followed by RANKL typically results in microscopically recognizable OCs 4 days later (data not shown). Expression of NFATc1, the master regulator of osteoclastogenesis (4), increased significantly 4 days after RANKL treatment, while TRAF3 protein levels decreased progressively (Figure 1A) and TRAF3 mRNA levels remained unchanged (Supplemental Figure 1A; supplemental material available online with this article; doi:10.1172/JCI66947DS1). We generated a TRAF3 retroviral expression vector by inserting a human TRAF3 cDNA fragment into a pMX-IRES-GFP retroviral expression vector. After transfecting pMX-IRES-GFP or pMX-TRAF3-IRES-GFP vectors into PlatE packaging cells using Fugene 6, virus supernatants were collected and added to WT OCPs generated by culturing BM cells with M-CSF for 3 days (20). Approximately 60\%-70\% infection efficiency was confirmed in all samples by flow cytometry (data not shown). GFP ${ }^{+}$cells were sorted and cultured with M-CSF plus RANKL. Numerous tartrate-resistant acid phosphatase-positive $\left(\mathrm{TRAP}^{+}\right)$OCs were observed in pMX-GFP-infected cells, but OC formation was decreased approximately 3 -fold in PMX-TRAF3IRES-GFP-infected cells (Figure 1B).

We next generated 2 new lines of mice with TRAF3 expression targeted to OC lineage cells: Traf 3 f/f; cathepsin $K^{\text {cre }}$ (which we call $\mathrm{C}-\mathrm{cKO})$ and Traf3ff; ;lysozyme $M^{\text {cre }}(\mathrm{L}-\mathrm{cKO})$ mice, and found that C-cKO BM cells cultured with M-CSF plus RANKL for 3 days formed $\mathrm{TRAP}^{+}$multinucleated OCs, but WT cultures did not (Supplemental Figure 1B). Although C-cKO and WT cells had formed similar numbers of OCs 2 days later, C-cKO OCs were larger, resulting in significantly increased OC area (Supplemental Figure 1B) and suggesting that TRAF3 negatively regulates osteoclastogenesis and perhaps fusion. We observed similar findings in cells from L-cKO mice (data not shown). L-cKO and C-cKO mice have normal bone phenotypes, similar to WT mice (Supplemental Figure 1C). Thus, we used Cre littermates as WT controls for future experiments. When we used RANKL at low concentrations $(\leq 5 \mu \mathrm{g} / \mathrm{ml})$, WT OCs generally formed later and were smaller (data not shown), but C-cKO cells in these conditions had greater OCforming ability than WT cells (Figure 1C) and increased resorption pit areas when cultured on bone slices with M-CSF plus RANKL for 9 days, although OC numbers were similar by this time (Supplemental Figure 2). We also cultured these cells on 6-well plates for 6 days, resuspended the cells with $0.25 \%$ trypsin/EDTA, and replated equal numbers of OCs on bone slices in 96-well plates for an additional 4 days. Resorption pit area and numbers were similar between C-cKO and WT OCs (data not shown), consistent with TRAF3 having its major role in RANKL-induced OC formation rather than function.

Importantly, we found that 2-month-old C-cKO mice have mild osteoporosis, as indicated by significantly reduced tibial trabecular bone volume (BV) (Figure 1D), trabecular number, and thickness, with mean values for trabecular connectivity and separation being numerically lower and higher, respectively, but these did not reach statistical significance (Supplemental Figure 1D). These were associated with increased OC numbers and surfaces (Figure 1E), compared with control mice.

Previous TRAF3 overexpression studies in B cells suggested that TRAF3 suppresses canonical NF- $\kappa B$ activation (21). To test the mechanism by which TRAF3 inhibits osteoclastogenesis, we examined canonical and noncanonical protein levels in OCPs overexpressing TRAF3 in response to RANKL and observed markedly increased expression of NFATc1, NIK, p52, RelB, and RelA in pMX-GFP-infected OCPs (Figure 1F). In RANKL-treated pMXTRAF3-IRES-GFP-infected OCPs, induction of NFATc1, NIK, RelA, and RelB was reduced compared with GFP-infected controls, accompanied by decreased p100 to p52 processing (Figure 1F). Consistent with these findings, we observed increased RelA, RelB, and NIK levels and increased p100 to p52 processing in L-cKO OCPs, even without RANKL stimulation (Figure 1G). Further addition of RANKL did not affect expression levels of these molecules, presumably reflecting prior exposure of primary cells to RANKL in vivo or the effects of constitutively active NIK. Furthermore, RelA nuclear translocation is increased in L-cKO OCPs under basal and RANKL-induced conditions (Figure $1 \mathrm{H}$ ), indicating enhanced NF- $\mathrm{KB}$ signaling. We observed similar findings in $\mathrm{C}-\mathrm{cKO}$ cells (data not shown).

TRAF3 ring finger domain is required for RANKL-induced TRAF3 degradation through a lysosome/autophagosome-dependent mechanism. TRAF 3 contains 3 major domains: a ring finger (RF), required for suppression of NIK (22); a zinc finger (ZF), required for NF- $\kappa$ B activation (23); and a TRAF domain, which binds degradationtargeted proteins, such as NIK (22). We overexpressed RANK and TRAF3 in 293 T cells to examine which TRAF3 domain or domains are required for its degradation. TRAF3 protein levels decreased significantly in these cells with serum starvation, which can induce autophagy in many cell types (24), and with RANKL treatment (Figure 2A). In contrast, TRAF3 accumulated with serum starvation or RANKL in cells expressing TRAF3 deletion mutants lacking either the RF or the RF and ZF domains. In contrast, with serum-starvation or RANKL treatment, TRAF3 levels decreased slightly in cells with the TRAF domain deleted. We observed similar findings in L-cKO OCPs infected with WT and mutant TRAF3 retroviruses. These findings contrast with a report that a NIK mutant lacking the TRAF3-binding domain is constitutively active (11). They suggest that the TRAF3-binding domain of NIK is necessary for NIK proteasomal degradation, while the TRAF domain of TRAF3 is not essential for TRAF3 degradation.

CD40 or BAFF-R engagement in B cells induces rapid, proteasome-dependent TRAF3 degradation (25). To determine whether RANKL induces TRAF3 ubiquitination and degradation in OCPs, we incubated endogenous TRAF3 proteins with a high-binding affinity ubiquitin matrix and captured ubiquitinated proteins using an anti-TRAF3 Ab. RANKL markedly increased TRAF3 ubiquitination (Figure $2 \mathrm{~B}$ ), which can occur in proteasomes, lysosomes, and autophagosomes in mammalian cells (26). To examine the mechanism involved, we pretreated WT OCPs with the lyso- 


\section{A}
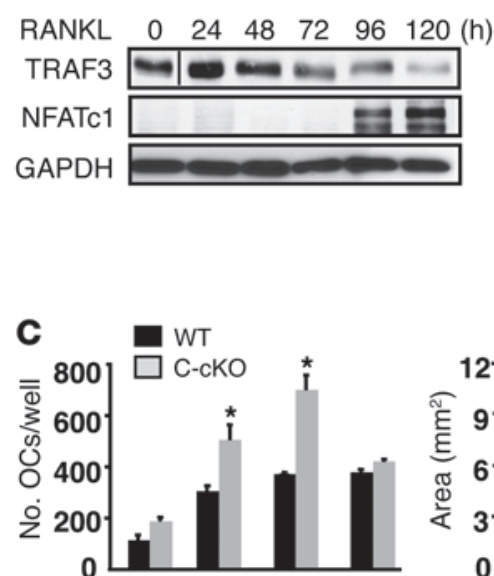

RANKL $1.25 \quad 2.5 \quad 5$ $(\mu \mathrm{g} / \mathrm{ml})$

E
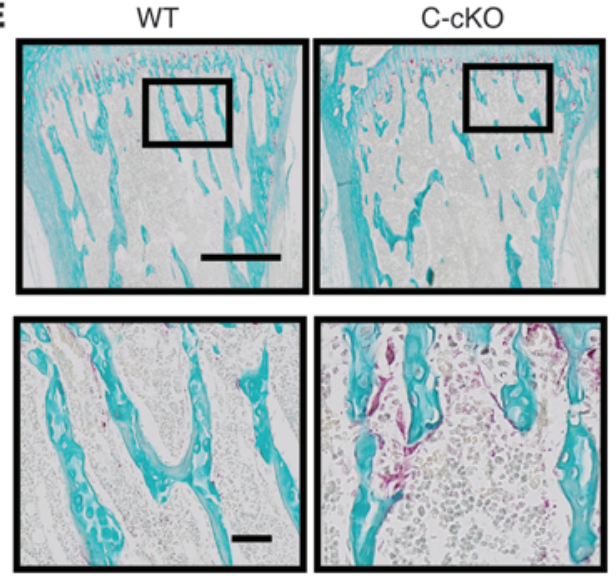

$\mathbf{F}$

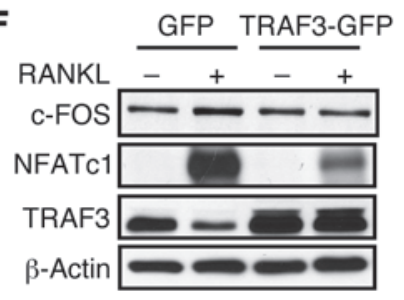

B

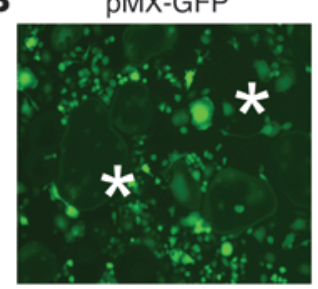

WT

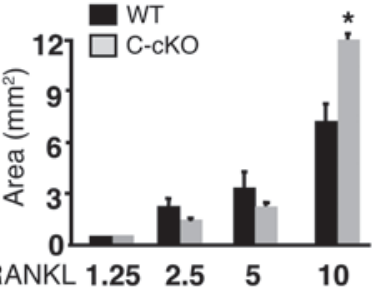

$(\mu \mathrm{g} / \mathrm{ml})$

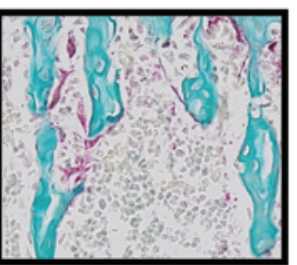

pMX-TRAF3-GFP

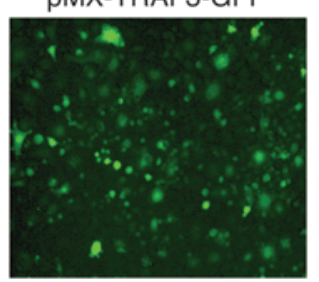

D

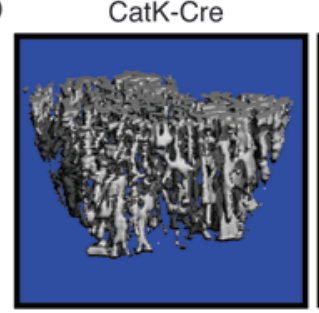

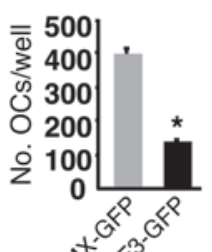

50 set

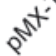

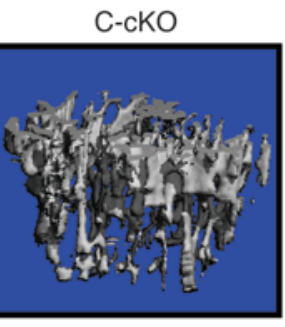
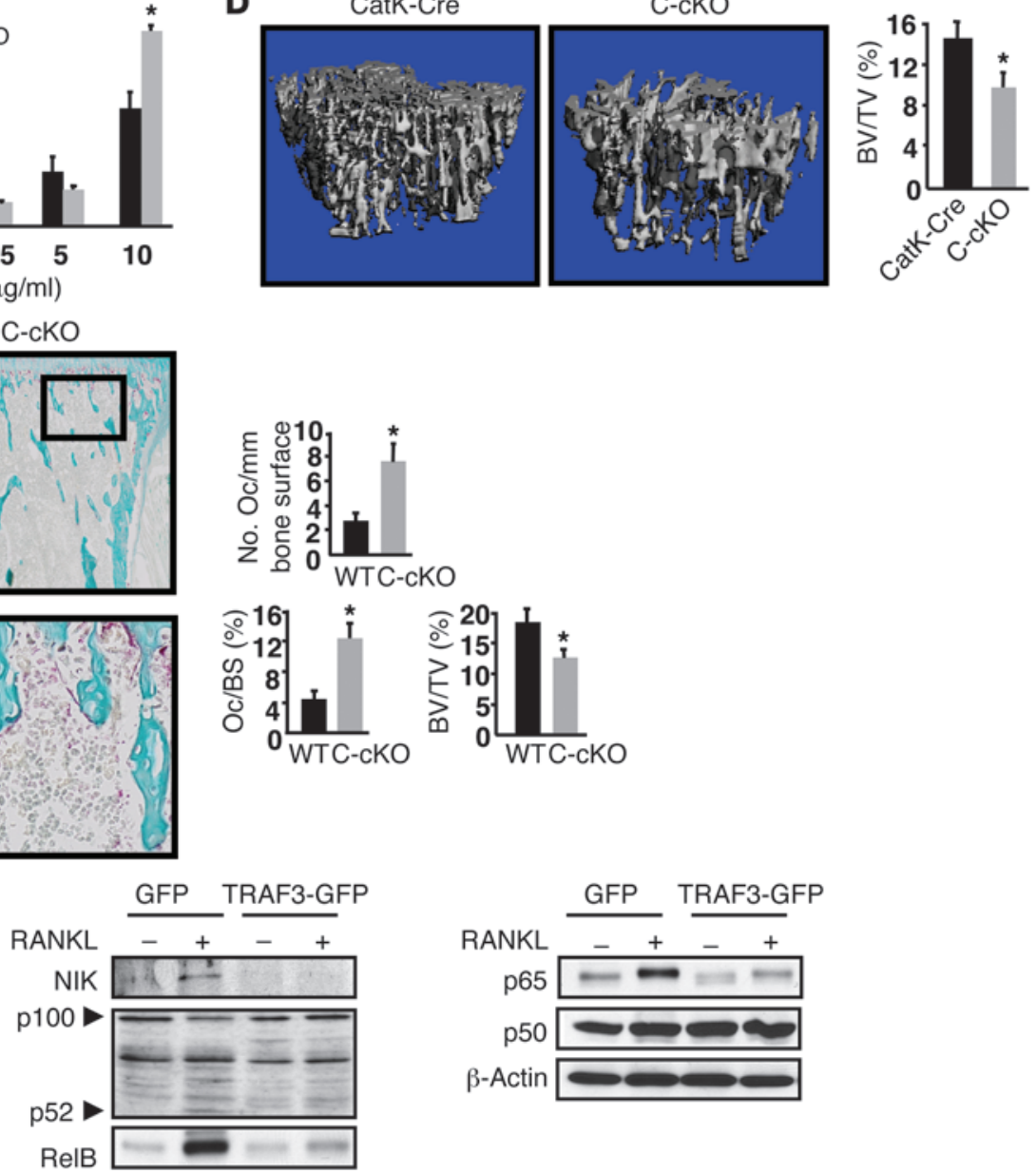

G
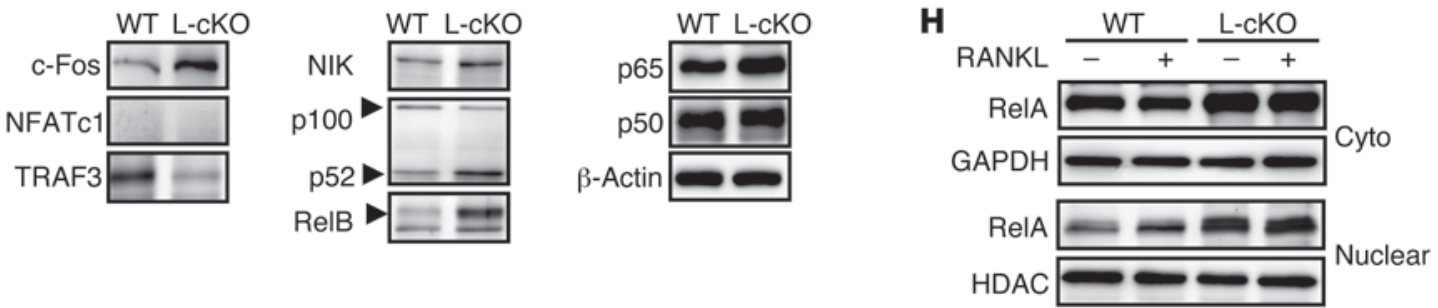

Figure 1

Mice with OC-specific deletion of TRAF3 have increased osteoclastogenesis and mild osteoporosis mediated by increased canonical and noncanonical NF-KB signaling. (A) WBs of TRAF3 and NFATc1 expression in WT BM cells treated with M-CSF plus RANKL. Lanes were run on the same gel, but were noncontiguous. (B) OCs formed from FACSAria-sorted WT OCPs infected with GFP or TRAF3-IRES-GFP (TRAF3-GFP) retroviruses treated with RANKL. Original magnification, $\times 10$. *OC centers. TRAP+ OCs were counted. (C) BM-derived C-cKO or WT cells cultured with RANKL. ${ }^{\star} P<0.05$. (D) Representative tibial $\mu \mathrm{CT}$ scans from 2-month-old CatK-Cre or C-cKO mice. ${ }^{*} P<0.05$. (E) Representative TRAP-stained tibial sections and bone histomorphometry from 2-month-old WT or C-cKO mice; boxed areas in lower images. Scale bars: 500 um (upper panels); 50 um (lower panels). OcS/BS, OC surface/bone surface (\%). ${ }^{*} P<0.05$. (F) WBs of whole cell lysates of WT OCPs infected with GFP or TRAF3-IRESGFP retroviruses and cultured with RANKL for 5 days. ( $G$ and $\mathbf{H}$ ) WBs of WT and L-cKO OCPs treated with RANKL for 48 hours. Cyto, cytoplasmic. 
A

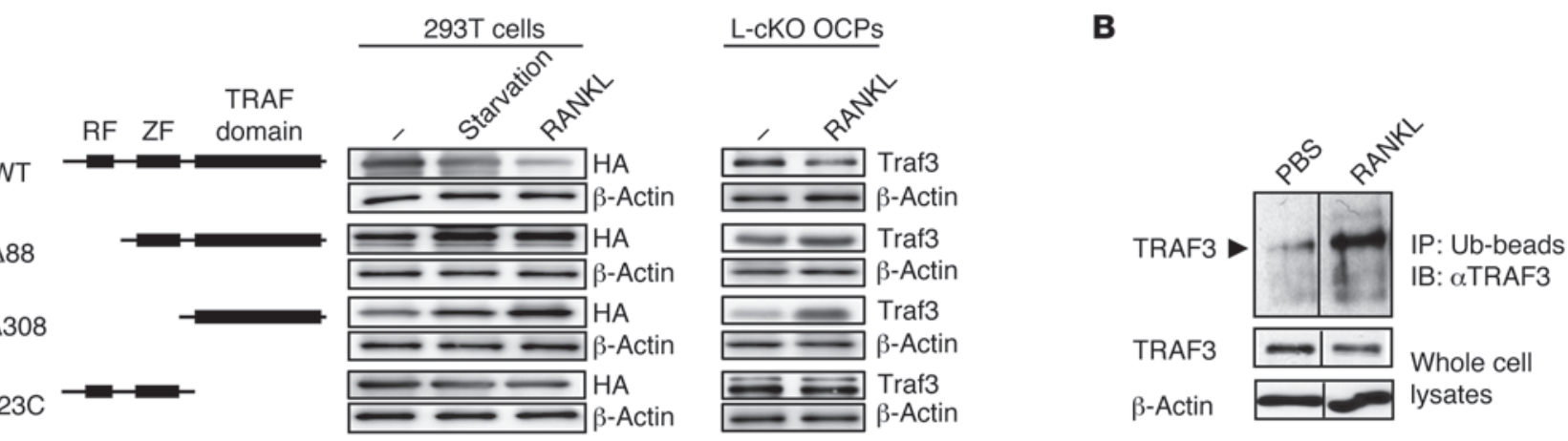

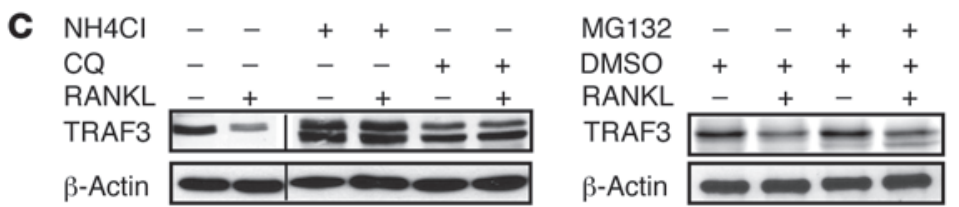

MG132 - $-\quad+\quad+\quad+$

DMSO + + + - - -

RANKL - 15'30' - 15' 30'

$\mathrm{IkB} \alpha \longrightarrow-\infty$

$\beta$-Actin

D Bafilo $+\quad+\quad-\quad-\quad-\quad-$

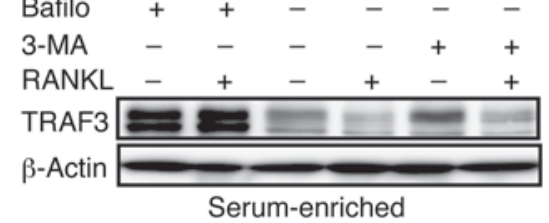

$\mathbf{F}$

\section{3-MA RANKL TRAF3 $\beta$-Actin} TRAF3-GFP

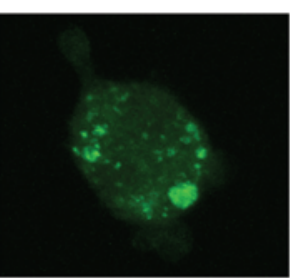

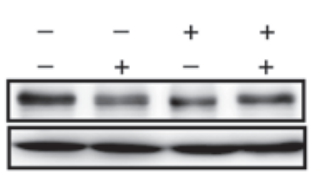

Serum-deprived Overlay
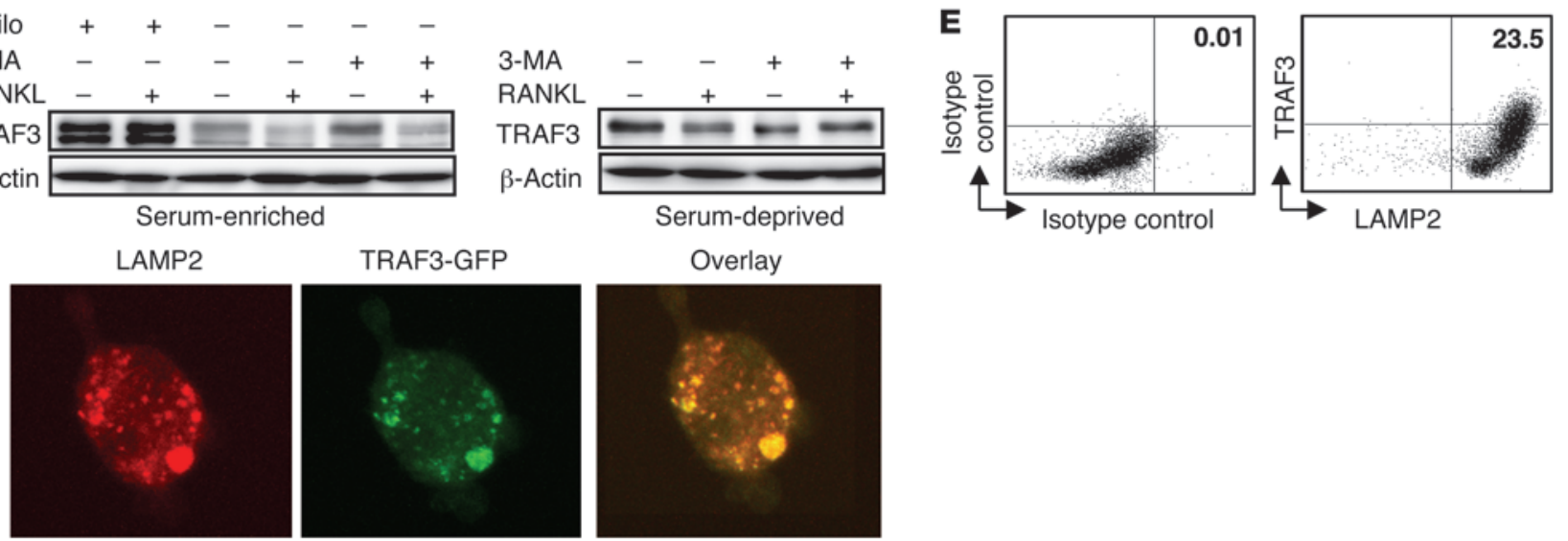

G RANKL CQ

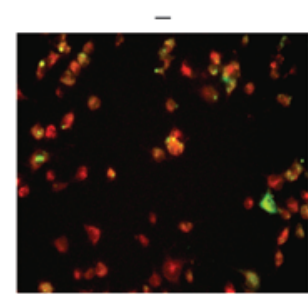

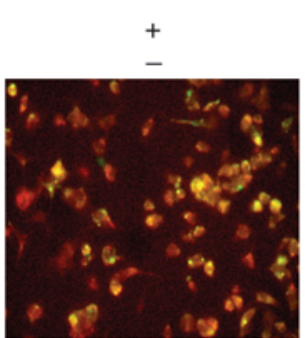
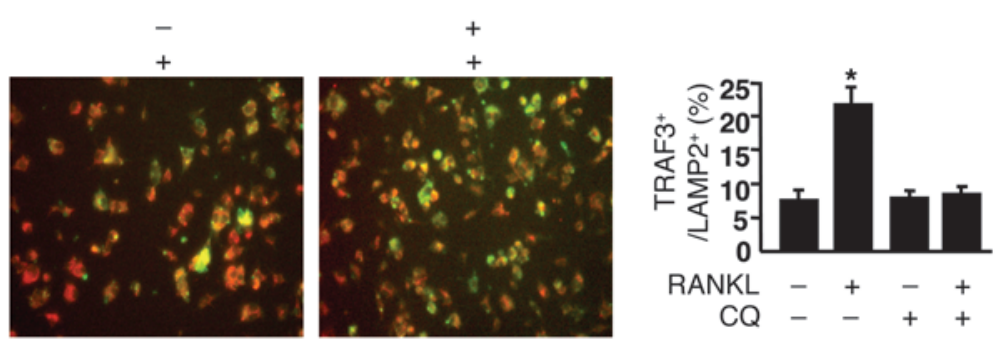

\section{Figure 2}

RANKL-induced TRAF3 degradation is lysosome mediated. (A) 293T cells transfected with HA-tagged WT or mutant TRAF3 constructs. L-cKO BM-derived OCPs infected with WT or mutant pMX-TRAF3 viruses were serum deprived or treated with RANKL for 8 hours. (B) Ubiquitinated (Ub) proteins from whole cell lysates of RANKL-treated (2 hours) WT OCPs using UbiQapture-Q Matrix blotted with anti-TRAF3 Ab. (C) WT OCPS pretreated with $\mathrm{NH}_{4} \mathrm{Cl}(50 \mathrm{mM})$ for 1 hour, $\mathrm{CQ}(50 \mathrm{mM})$ for 6 hours, or MG132 $(20 \mathrm{mM})$ for 4 hours were treated with RANKL for 8 hours. For IкB $\alpha$, WT OCPs were treated with DMSO or MG132 with or without RANKL for indicated times. Lanes were run on the same gel, but were noncontiguous in B and C. (D) WT OCPs pretreated with bafilomycin (Bafilo; $50 \mathrm{ng} / \mathrm{ml}$ ) for 16 hours or 3-MA (3-MA (5 mM) for 2 hours were treated with or without RANKL for 8 hours in serum-enriched or -deprived conditions. (E) TRAF3-GFP retrovirus-infected WT OCPs treated with RANKL for 8 hours were fixed and double-stained with TRAF3 and LAMP2 Abs. Left plot shows background staining with isotype control Abs. (F) TRAF3GFP retrovirus-infected WT OCPs stained with anti-LAMP2 Ab. Colocalization assessed using confocal microscopy. Original magnification, $\times 60$. (G) TRAF3-GFP retrovirus-infected WT OCPs treated with or without RANKL or CQ (2 mM) were stained with anti-LAMP2 Ab. TRAF3/LAMP2 double-positive cells were counted. Original magnification, $\times 20 .{ }^{*} P<0.05$.

some inhibitors chloroquine (CQ) for 6 hours or $\mathrm{NH}_{4} \mathrm{Cl}$ for 1 hour or with the proteasome inhibitor MG132 for 4 hours, followed by RANKL, and evaluated the extent of TRAF3 degradation by Western blot (WB). Pretreatment with either CQ or $\mathrm{NH}_{4} \mathrm{Cl}$, but not MG132, markedly increased TRAF3 levels in response to RANKL
(Figure 2C). To confirm that MG132 functioned effectively as a proteasome inhibitor (27), we treated WT OCPs with RANKL plus MG132 for 15 and 30 minutes and found that compared with DMSO control, MG132 blocked RANKL-induced IкB $\alpha$ degradation (Figure 2C). 
A

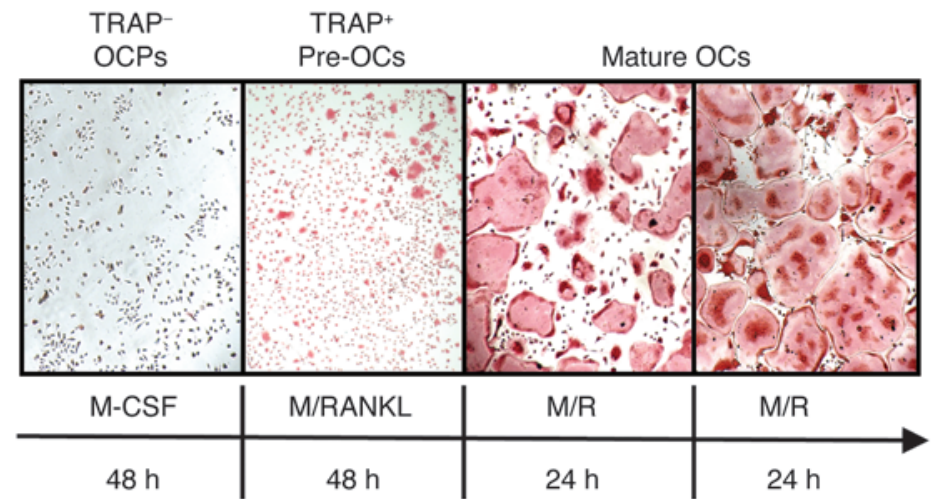

Proliferation

Differentiation

Fusion +

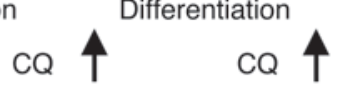

Figure 3

CQ inhibits OC formation in vitro. (A) Typical in vitro OC formation time-course. BM-derived WT OCPs were treated with M-CSF (M) for 2 days and with M-CSF plus RANKL (R) for 4 days. CQ was added on day 2 (B), or day 4 (C). Original magnification, $\times 4 .{ }^{*} P<0.05$. (D) WBs of WT OCPs treated with RANKL with or without CQ $(10 \mu \mathrm{M})$ for 3 days. (E) WBs of Traf3 $3^{t / f}$ OCPs infected with MSCV-GFP (GFP) or MSCV-CreGFP (Cre-GFP) retroviruses and GFP-FACS sorted or $(\mathbf{F})$ cultured with RANKL with or without CQ $(2 \mu \mathrm{M})$ for 5 days. (G) WBs of whole cell lysates from WT or TRAF3 L-cKO mouse tissues. (H) WBs of BM cells cultured with RANKL with or without CQ $(2 \mu \mathrm{M})$ for 5 days. ${ }^{\star \star} P<0.01$ vs. PBS.

B
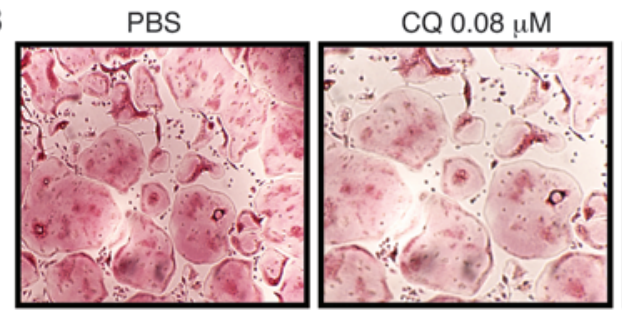

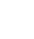

$\mathrm{CQ} 0.4 \mu \mathrm{M}$
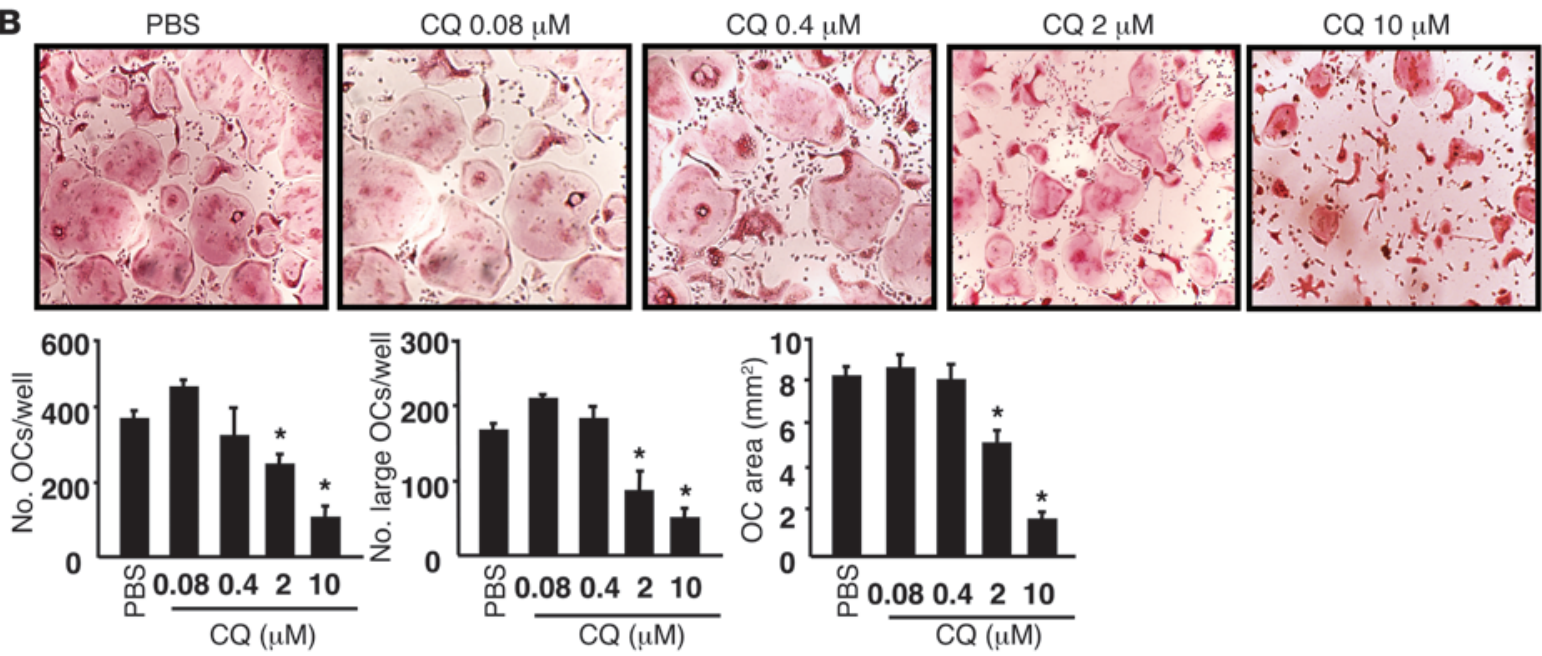

C
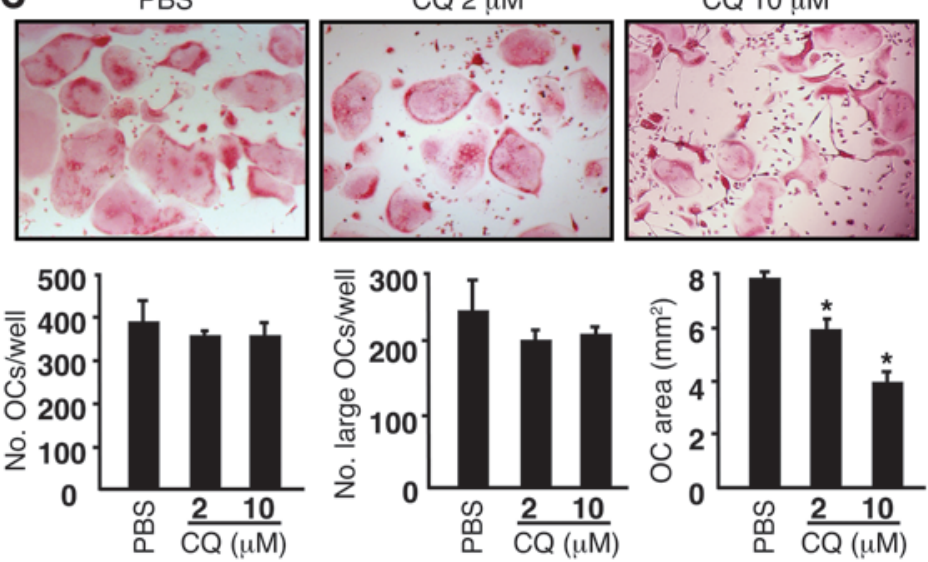

D

F

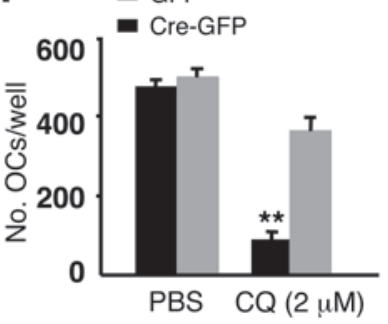

G

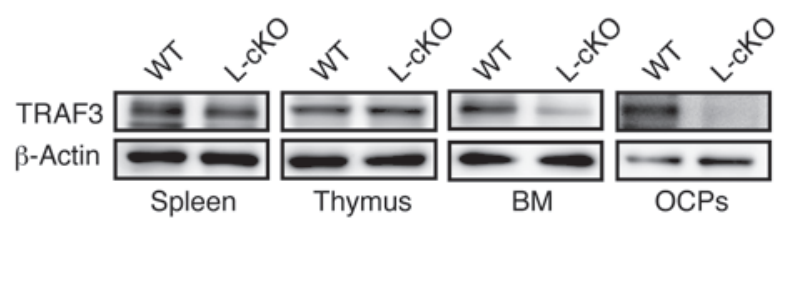

E - - - GFP
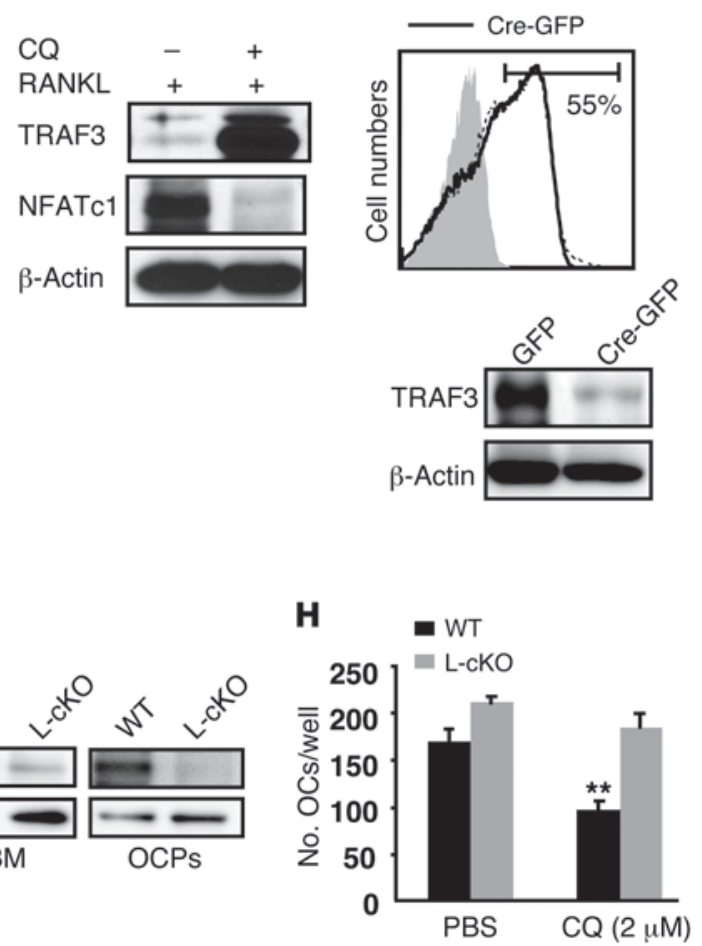
A
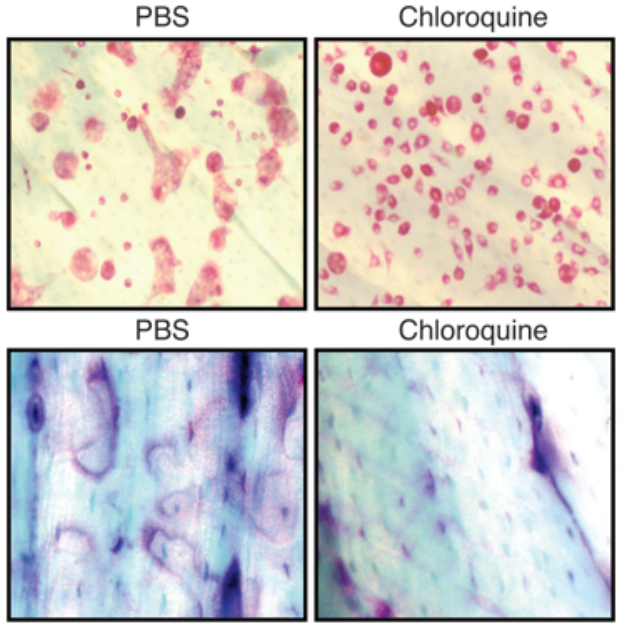

B
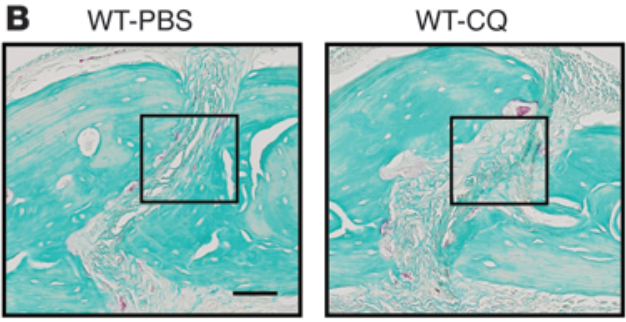

C WT-PBS
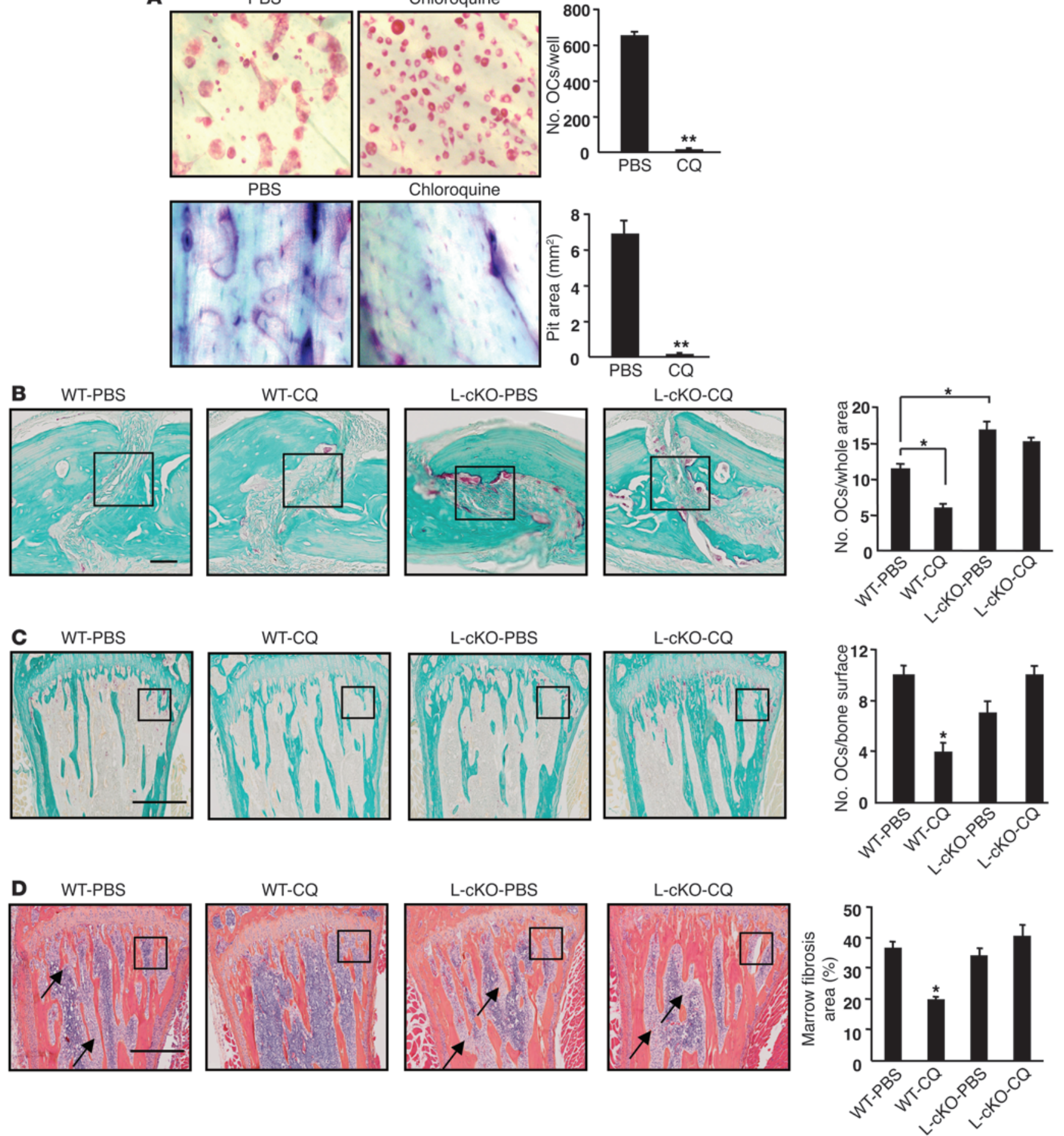

Figure 4

CQ affects OC function in vitro and in vivo. (A) WT OCPs cultured with RANKL with or without CQ (5 $\mu$ M) for 9 days. TRAP staining (left panels) and toluidine blue (right panels, to highlight resorption pits). Values are means + SEM of 4 wells. ${ }^{* *} P<0.01$ vs PBS. Original magnification, $\times 20$. (B-D) Representative TRAP-stained sections and OC numbers in calvarial (B) and tibial (C) sections from 10- to 12-week-old male WT or L-cKO mice treated with CQ $(50 \mathrm{mg} / \mathrm{kg} / \mathrm{d}$ i.p. for 10 days) and given supracalvarial injections of hPTH(1-34 aa) (10 $\mu \mathrm{g} / \mathrm{mouse}) 4 \times / \mathrm{d}$ for 3 days beginning on day 7. (D) Representative H\&E-stained tibial sections from the mice in $\mathbf{C}$ illustrating marrow fibrosis (arrows) and values for percentage of marrow space occupied by marrow fibrosis. Values are means + SEM of 4 mice/group. ${ }^{*} P<0.05$. Boxed areas in $\mathbf{B}-\mathbf{D}$ are illustrated at higher magnification in Supplemental Figure 5. Scale bars: $50 \mu \mathrm{m}$ (B); $500 \mu \mathrm{m}$ (C and D). 

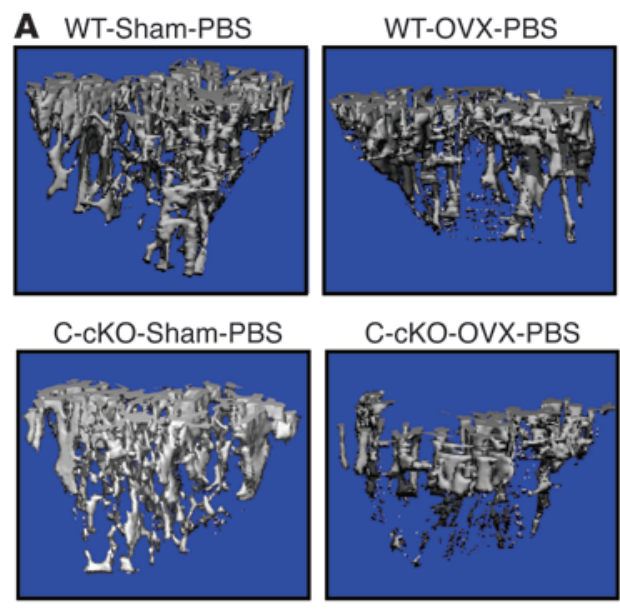

B WT-Sham-PBS
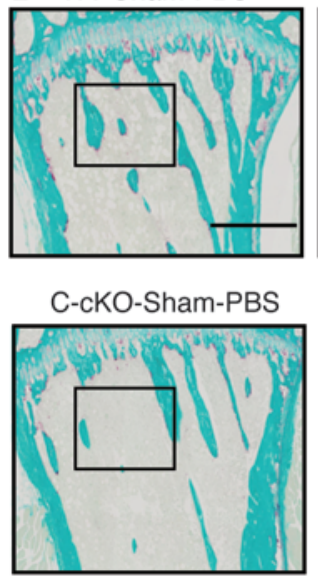
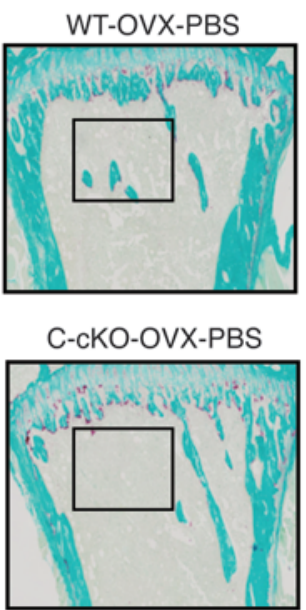
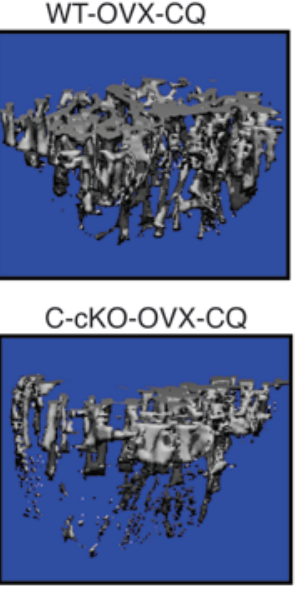

WT-OVX-CQ

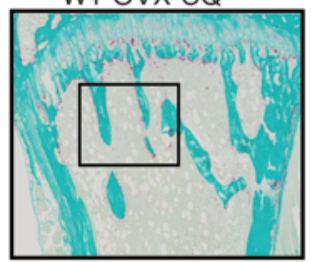

C-cKO-OVX-CQ

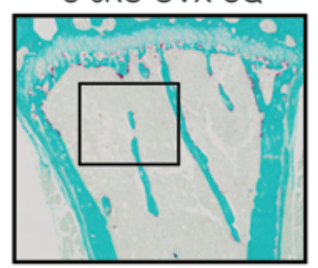

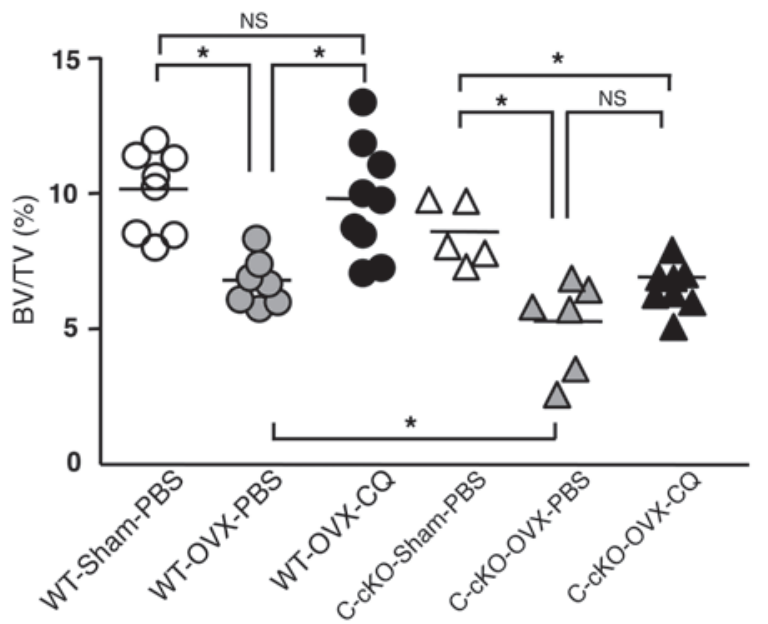
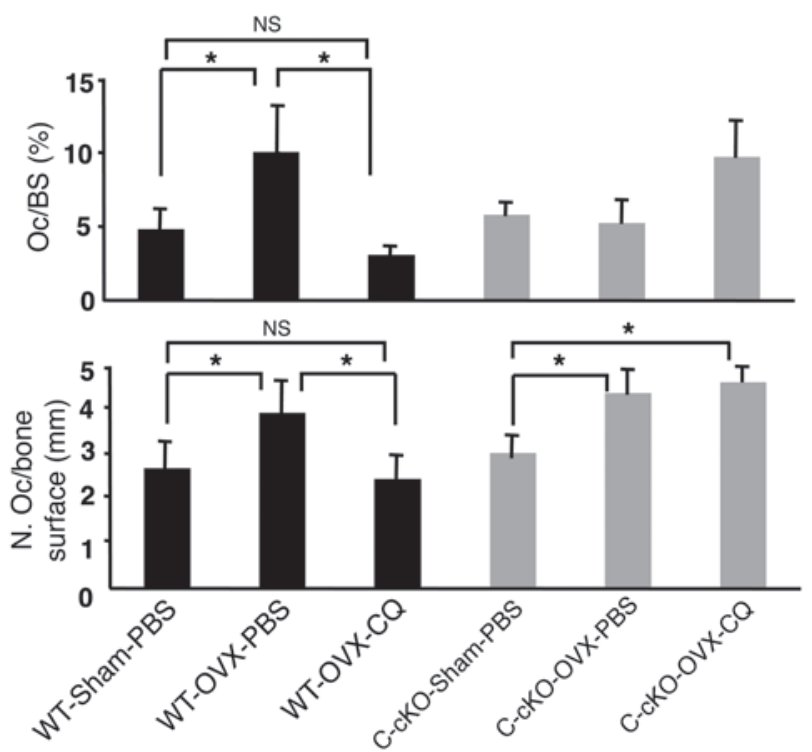

Figure 5

CQ prevents OVX-induced bone loss in WT, but not in C-cKO mice. OVX or sham-operated 8- to 9-week-old WT and C-cKO mice treated with PBS or CQ (50 mg/kg/d i.p. for 28 days). (A) Representative $\mu$ CT images of tibiae and trabecular BVs. (B) Representative TRAP-stained tibial sections and histomorphometric data for OC surface and numbers. Values are the mean + SEM of 5 9 mice/group. ${ }^{*} P<0.05$. Boxed areas are shown at higher magnification in Supplemental Figure 6B. Scale bar: $500 \mu \mathrm{m}$.

We next tested autophagy inhibitors on RANKL-induced TRAF3 degradation. Bafilomycin A1 blocks fusion of the autophagosome and the lysosome (28); it increased accumulation of TRAF3 (Figure 2D). 3-Methyladenine (3-MA), which blocks PI3K, had no effect on TRAF3 degradation in serum-enriched conditions. However, TRAF3 accumulated in serum-deprived OCPs treated with 3-MA (Figure 2D), consistent with 3-MA-inhibiting autophagy only in serum-deprived cells (29). We next infected WT OCPs with a PMX-TRAF3-GFP retrovirus and found that TRAF3 and LAMP2 (a lysosome marker) were coexpressed in approximately $23 \%$ of RANKL-treated cells (Figure 2E). Confocal microscopy showed that the TRAF3-GFP fusion protein was localized mostly in the cytosol in OCPs (Figure 2F). RANKL significantly increased colocalization of TRAF3 and LAMP2, which was reduced by CQ (Figure 2G).

CQ inhibits RANKL-induced OC formation by preventing lysosomal degradation of TRAF3. To determine whether CQ affects OC formation, we cultured WT BM cells with M-CSF for 2 days, added RANKL, and stained them for TRAP activity on days 2, 4, 5, and 6 . Two days after RANKL addition, OCPs differentiated into $\mathrm{TRAP}^{+}$mononuclear cells, which we called pre-OCs, that ultimately fused to form OCs over the following 2 days (Figure 3A). When we added CQ during the initial stages of OC formation, the total number of OCs and large OCs and OC area were significantly reduced dose dependently, with the highest dose $(10 \mu \mathrm{M})$ inducing a 3-fold reduction in OC numbers (Figure 3B). Addition of CQ during the later stages of OC formation reduced OC area, with no effect on total and large OC numbers (Figure 3C). Furthermore, when these OCPs were treated with RANKL plus CQ for 3 days, the percentage of apoptotic and dead cells was similar to that in controls (6.1 vs. $5.3 \%$, data not shown). Thus, CQ inhibits OCP differentiation, but not OCP fusion or OC survival.

$\mathrm{CQ}$ is an anti-malarial agent, and like its derivative, hydroxychloroquine, has been used early in the treatment of systemic lupus 
A

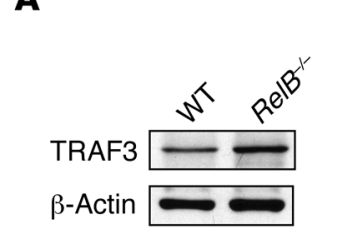

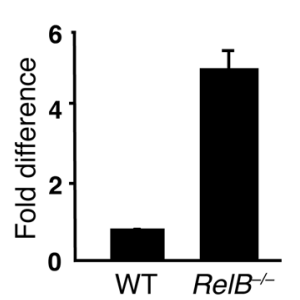

B

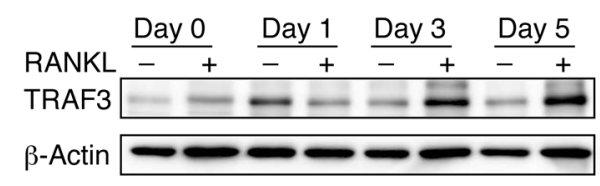

C
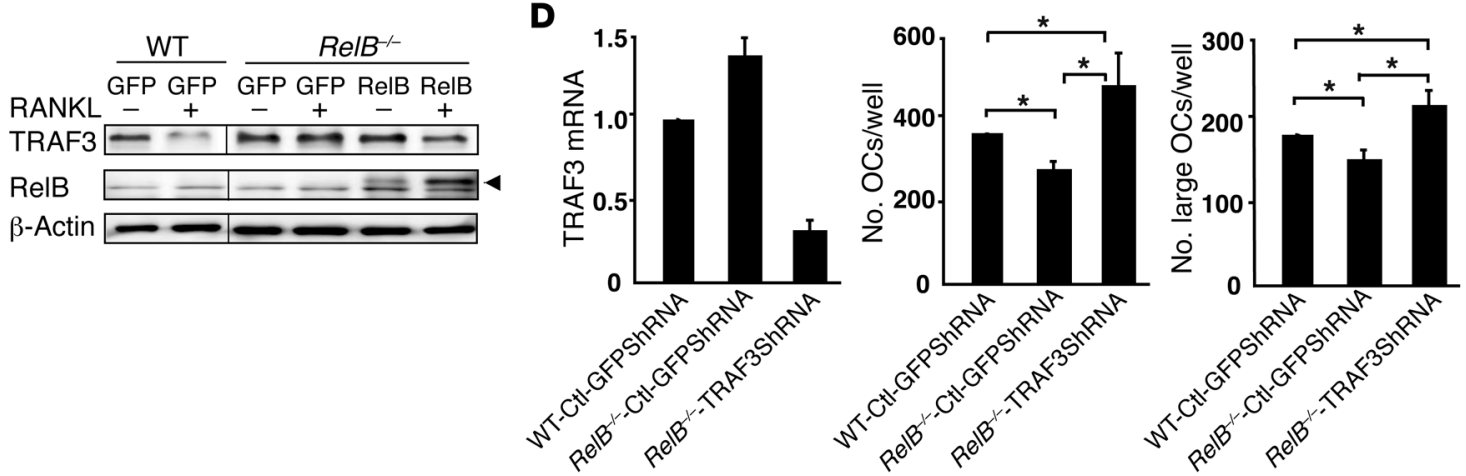

$\mathbf{E}$
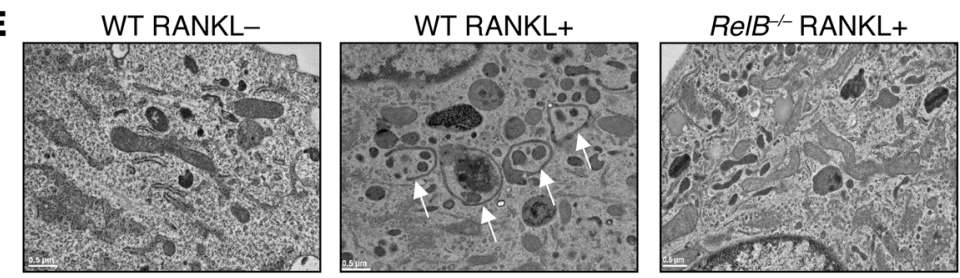

Figure 6

RelB positively regulates RANKL-induced TRAF3 degradation. (A) WBs of WT and Re/B-/- BM whole cell lysates and TRAF3 levels quantified densitometrically (mean + SEM of 3 blots). (B) WBs of RANKL-treated Re/B ${ }^{-1-}$ OCPs. (C) WBs of WT or Re/B ${ }^{-/}$OCPs infected with pMY-GFP or pMY-RelB-GFP retrovirus for 2 days and treated with RANKL for 8 hours. Arrowhead, specific RelB band. Lanes were run on the same gel, but were noncontiguous. (D) WT or RelB-/- OCPs infected with control GFP shRNA (Ctl-shRNA) or TRAF3 shRNA lentiviruses for 2 days were cultured with MCSF plus RANKL for 4 days. TRAF3 knockdown was confirmed by RT-PCR. Total numbers of OCs and large OCs were counted. (E) EM images of WT or RelB-/- OCPs treated with RANKL for 4 days in 2-well culture chambers showing autophagosomes (arrows).

erythematosus and RA, but the precise mechanism of action in autoimmune diseases is not clear (30). We found that TRAF3 levels increased markedly in OCPs after CQ treatment (10 $\mu \mathrm{M}$ for 3 days); this was accompanied by decreased NFATc1 expression (Figure 3D). We infected OCPs from Traf3f/f mice with MSCV-Cre-GFP (where MSCV indicates murine stem cell virus) or MSCV-GFP retrovirus and sorted $\mathrm{GFP}^{+}$cells to verify deletion of TRAF3 (Figure 3E). Sorted $\mathrm{GFP}^{+}$cells then were cultured with or without CQ in OC formation assays. TRAF3-deficient and WT OCPs formed similar numbers of OCs, and CQ reduced OC numbers from WT cells $(\sim 80 \%)$, but had no effect on TRAF3-deficient cells (Figure 3F). We next confirmed specific deletion of TRAF3 protein in BM cells and OCPs from L-cKO mice (Figure 3G) and found that CQ did not inhibit RANKLinduced OC formation in TRAF3-deficient cells (Figure $3 \mathrm{H}$ ).

CQ inhibits OC formation in vitro and $P T H$ - and ovariectomy-induced bone resorption in vivo via TRAF3. We next cultured WT OCPs on bone slices with RANKL for 9 days and found that CQ-treated cells formed few TRAP ${ }^{+}$OCs ( $12 \pm 3$ vs. $656 \pm 17$ in controls) and few resorption pits, which covered very little of the bone slices $\left(0.04 \pm 0.01 \mathrm{~mm}^{2}\right)$ compared with controls where pits occupied most of the surface $\left(6.9 \pm 0.7 \mathrm{~mm}^{2}\right)$ (Figure 4A). We then treated 3 -week- or 3-month-old WT and C-cKO mice with CQ $(25 \mathrm{mg} / \mathrm{kg}$ i.p, once daily) for 28 days and found no significant difference in $\mathrm{BV}$ or other structural parameter values assessed by $\mu \mathrm{CT}$ in tibiae compared with those of vehicle-treated mice (Supplemental Figure 3, A and B), suggesting that this regimen of CQ has no effect on basal bone resorption or bone mass. Further time-course and dose-response studies will be required to determine whether CQ can inhibit basal bone resorption.

To determine whether CQ affects osteoblast formation or function, we performed CFU-F, CFU-alkaline phosphatase (CFU-ALP), and bone nodule formation assays using $2 \mu \mathrm{M} \mathrm{CQ}$, which inhibits OC formation. We found that CQ had no effects on any of these indices of osteoblast function in WT BM stromal cells (Supplemental Figure 4A), suggesting that the predominant effect of CQ is on OCs. Parathyroid hormone (PTH) is a primary regulator of calcium homeostasis and has both anabolic and catabolic effects, associated with intermittent or continuous administration, respectively $(31,32)$. To test whether CQ affects PTH-induced bone formation, we administered PTH $(2 \mu \mathrm{g} / \mathrm{kg}, 3 \times / \mathrm{d})$ intermittently to WT mice for 14 days along with CQ $(50 \mathrm{mg} / \mathrm{kg}, 1 \times / \mathrm{d})$. PTH treatment significantly increased trabecular BV, trabecular thickness, and mineral apposition (MAR) and bone formation rates (BFR), but CQ had no effect (Supplemental Figure 4, B-D). 


\section{A}

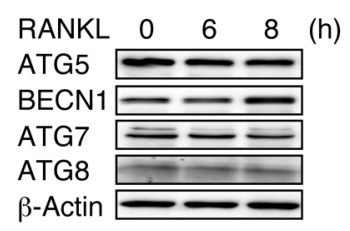

B

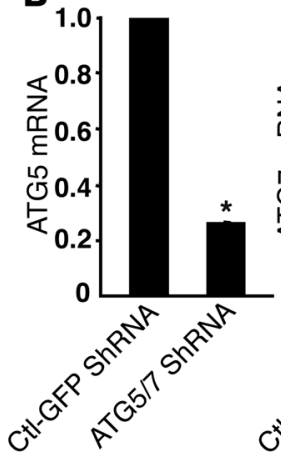

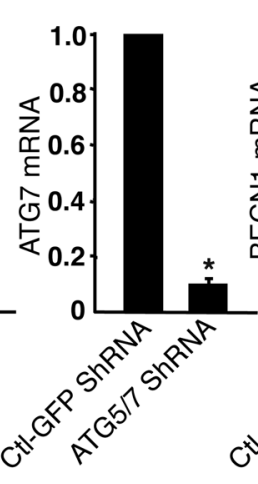

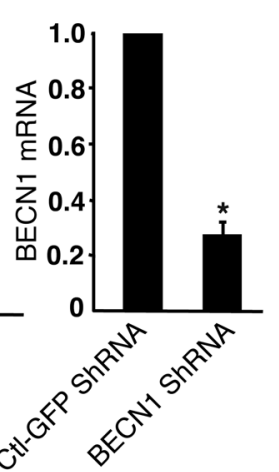

C

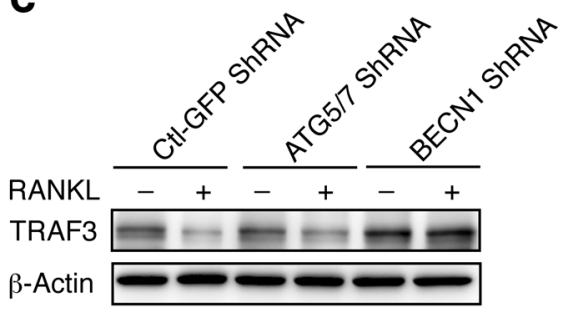

D

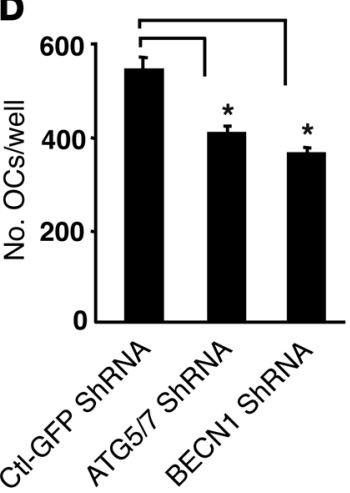

E

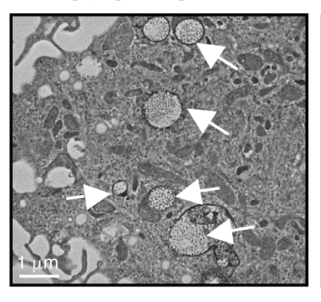

ATG5/7 ShRNA

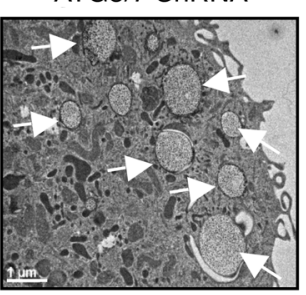

BECN1 ShRNA

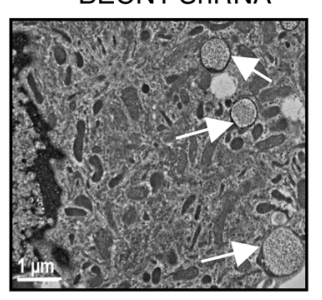

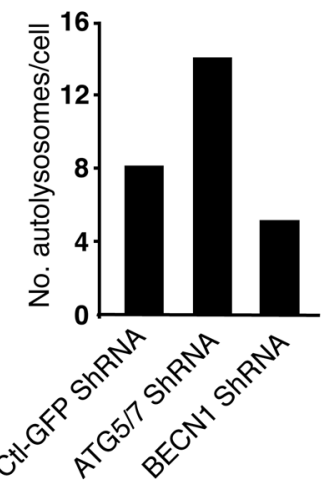

Figure 7

RANKL-induced TRAF3 degradation is absent in BECN1, but not in ATG5/7 knockdown OCPs. (A) WBs of WT OCPs treated with RANKL. (B-E) WT OCPs infected with Ctl-GFP, BECN1, or ATG5 and ATG7 shRNA lentiviruses for 2 days. (B) mRNA levels of BECN1, ATG5, and ATG7. (C) WB of infected cells treated with RANKL for 8 hours. (D) OCs formed from infected cells cultured with RANKL for 5 days. ${ }^{*} P<0.05$ vs. Ctl-GFP shRNA. (E) EM images and numbers of autophagosomes (arrows, expressed/cell in at least 50 cells/preparation from 2 independent experiments) in infected cells.

We next examined the effects of CQ in an in vivo model of pathologic bone resorption in which PTH stimulates OC formation by inducing RANKL expression (33). We treated L-cKO or Cre-negative control mice with CQ $(50 \mathrm{mg} / \mathrm{kg}, 1 \times / \mathrm{d})$ for 7 days followed by CQ plus PTH (10 $\mu \mathrm{g} /$ mouse, $4 \times / d$, s.c. over calvariae) for another 3 days (34). CQ markedly reduced PTH-induced OC formation in calvariae and tibiae in WT mice compared with PBS treatment, but not in L-cKO mice (Figure 4, B and C, and Supplemental Figure 5 , A and B). A similar pattern of change was observed in serum TRAP5b levels (Supplemental Figure 5D). Furthermore, marrow fibrosis (a hallmark of PTH-induced resorption) was observed in all groups except the WT mice treated with CQ and PTH (Figure 4D and Supplemental Figure 5C). Similar results were obtained in a separate experiment using C-cKO mice (data not shown).

To further evaluate the effects of CQ on bone resorption in vivo, we used 8- to 9-week-old female C-cKO or Cre-negative control mice in an ovariectomy (OVX) model. Two days after surgery, mice were treated with PBS or CQ $(50 \mathrm{mg} / \mathrm{kg}$, i.p. $1 \times / \mathrm{d})$ for 28 days, and tibiae were analyzed using $\mu \mathrm{CT}$ and histology. Four weeks after surgery, uterine weights and tibial BVs in OVX mice were markedly reduced compared with those of sham-operated WT and C-cKO mice, consistent with OVX, and BVs were significantly lower in C-cKO than in WT mice (Figure 5A and Supplemental Figure 6A). In addition, CQ prevented OVX-induced bone loss and increased OC numbers and surfaces in WT mice, but not in $\mathrm{C}$-cKO mice (Figure 5, $A$ and B, and Supplemental Figure 6, B and C).
RelB is required for RANKL-induced TRAF3 lysosomal degradation by regulating $B E C N 1$ expression. We next investigated basal and inducible TRAF3 levels in RelB ${ }^{-/}$OCPs, in part because RANKL/NIK/ p100 signaling activates RelB (5) and also to determine whether RelB regulates TRAF3 degradation. Basal TRAF3 protein levels were 5-fold higher in RelB ${ }^{-1-}$ BM cells than in WT cells (Figure 6A). In addition, in contrast to the time-dependent decrease in TRAF3 levels induced by RANKL in WT OCPs (Figure 1A), significant accumulation of TRAF3 protein was observed in RANKL-treated $\mathrm{RelB}^{-/-}$OCPs (Figure 6B), suggesting that RelB promotes RANKLinduced TRAF3 degradation. To test this, we infected $\mathrm{RelB}^{-/-}$ OCPs with pMY-RelB to determine whether adding back RelB could restore RANKL-induced TRAF3 degradation. TRAF3 levels decreased significantly in GFP control virus-infected OCPs 8 hours after RANKL stimulation (similar to Figure 2C). This decrease did not occur in GFP control virus-infected $\mathrm{RelB}^{-/-} \mathrm{OCPs}$, but was seen in $\mathrm{RelB}^{-/-}$OCPs overexpressing pMY-RelB (Figure 6C).

M-CSF plus RANKL-treated RelB $^{-/-} \mathrm{BM}$ cells generated significantly fewer mature OCs than WT cells (8). To further investigate the role of TRAF3 in these conditions, we infected WT and RelB ${ }^{-/-}$ OCPs with control GFP (Ctl-GFP) or TRAF3 shRNA lentivirus and confirmed TRAF3 knockdown by RT-PCR. TRAF3 mRNA levels were $35 \%$ higher in $\mathrm{RelB}^{-/-}$than in control OCPs (Figure 6D), and $\mathrm{RelB}^{-/-}$ cells formed fewer OCs than WT cells. TRAF3 knockdown induced significantly more OCs from RelB $^{-/-}$cells $(267 \pm 18$ to $490 \pm 54)$ and to a greater extent than WT virus-infected cells (367 \pm 6 ; Figure $6 \mathrm{D})$. 
A

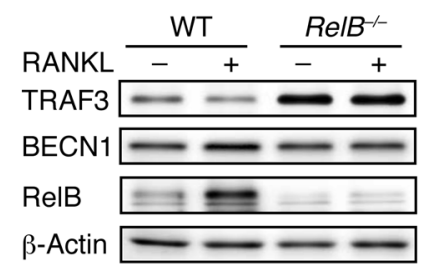

B

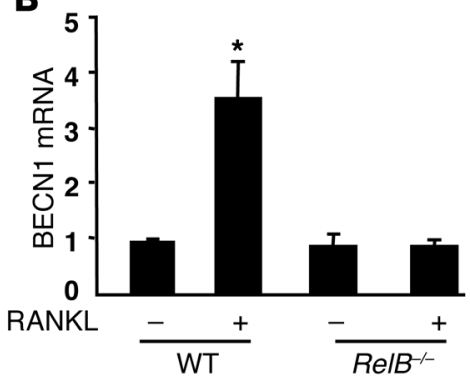

C

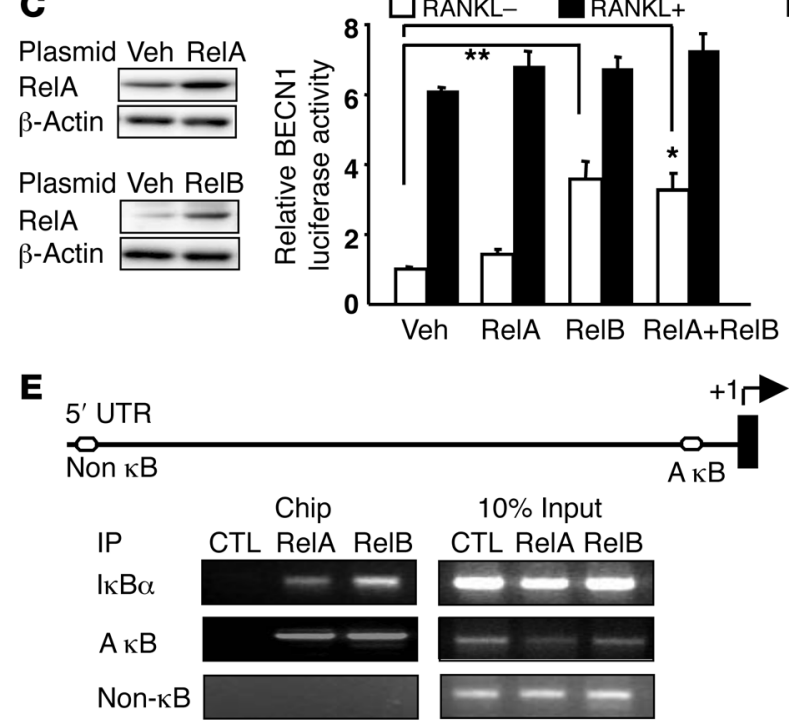

D
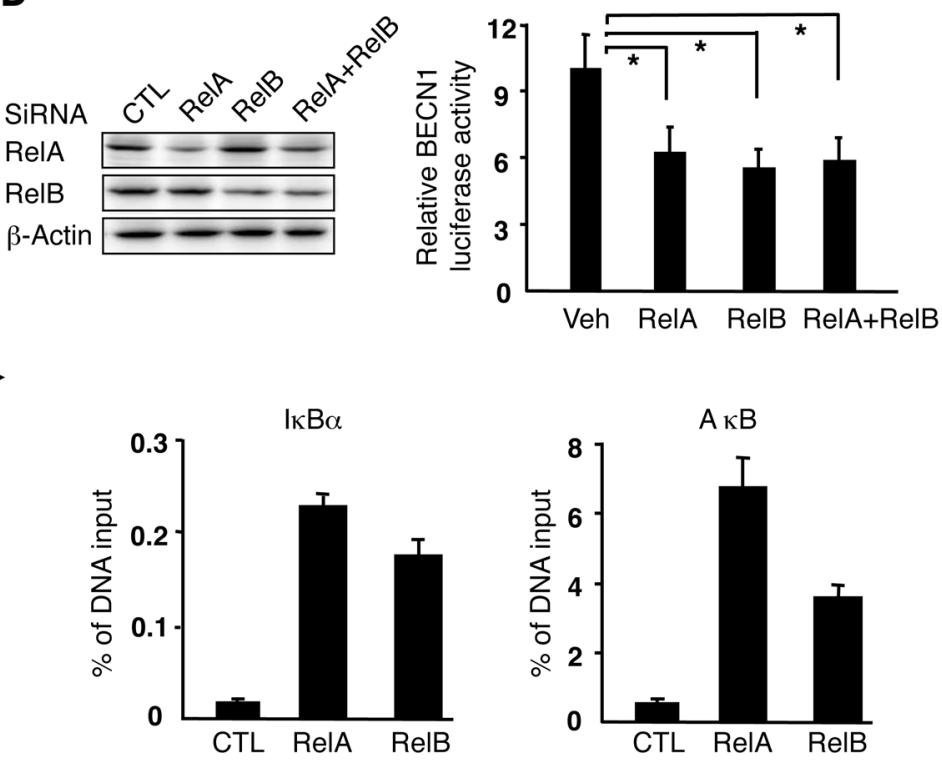

Figure 8

RelB binds to the BECN1 promoter and regulates its expression. Whole cell lysate WBs (A) and RT-PCR-detected BECN1 mRNA levels (B) in WT or RelB ${ }^{-1-}$ OCPs treated with RANKL or vehicle for 8 hours. (C) 293T cells transfected with RelA- or RelB-expressing plasmids or cotransfected with a human BECN1 promoter pGL3-Luc reporter and a Renilla luciferase plasmid and RelA- and/or RelB-expressing plasmids. RANKL-treated samples were cotransfected with a hRANK plasmid. (D) RelA- or RelB siRNAs were transfected into $293 \mathrm{~T}$ cells 48 hours before cotransfection with pGL3-Luc and Renilla plasmids. Protein levels were assessed by WB. Dual-luciferase assays were performed 24 hours after transfection. Values in $\mathbf{C}$ and $\mathbf{D}$ are means + SEM from 3 independent experiments. ${ }^{*} P<0.05$; ${ }^{*} P<0.01$. (E) WT OCPs treated with RANKL for 8 hours and sheared chromatin precipitated with RelA- or RelB-specific Abs, or IgG (negative control [CTL]). Recovered DNA was used as a template for PCR. Primers for the $A \kappa B$ site inside the $\mathrm{I}_{\mathrm{B}} \alpha$ promoter and a non- $\mathrm{K} B$ site in the proximal $\mathrm{BECN1}$ promoter region were positive and negative controls, respectively. RelA or RelB binding to the indicated promoters was quantified by real-time PCR. Data are representative of 2 independent experiments.

To further explore the role of RelB in RANKL-induced TRAF3 lysosomal degradation, we used transmission EM (widely used to monitor autophagy) (35) to evaluate the ability of WT and RelB-/cells to form autophagosomes. Initial steps in autophagy include formation and expansion of an isolation membrane, also called a phagophore, the edges of which fuse to form an autophagosome, a double-membraned vesicle that sequesters cytoplasmic material. Autophagosomes then fuse with lysosomes to form autolysosomes where the captured material, together with the inner membrane is degraded (36). Autophagic vacuoles with their characteristic double membranes formed in WT, but not in $\mathrm{RelB}^{-/-}$, cells in response to RANKL (Figure 6E).

Having demonstrated that RANKL-induced TRAF3 degradation is lysosome/autophagy-mediated, we next examined protein levels in OCPs of ATG5, ATG7, ATG8, and beclin 1 (BECN1), key molecules in autophagosome formation (37). RANKL upregulated expression of BECN1 ( 3 -fold), but not of the other autophagic pro- teins (Figure 7A). Moreover, RANKL-induced TRAF3 degradation and autolysosome formation were impaired in cells with shRNA knockdown of BECN1, but not of ATG5/7 (Figure 7, B, C, and E), associated with a significant reduction in OC numbers (Figure 7D). TRAF3 levels in OCPs treated with ATG5/7 shRNA fell in response to RANKL, similar to control shRNA, but this was associated with reduced osteoclastogenesis and increased autolysosome numbers, suggesting that ATG5/7 may regulate the degradation of other modulators that contribute to RANKL-induced OC formation.

BECN1 associates with PI3KIII/Vps34 as a platform that modulates autophagosome formation during early steps of autophagy (37). The reduction in TRAF3 induced by RANKL was accompanied by increased RelB and BECN1 protein and mRNA levels in WT OCPS, but not in RelB $^{-/-}$OCPs (Figure 8, A and B), suggesting an association between RelB and BECN1. There are $4 \mathrm{\kappa B}$ binding sites in the BECN1 promoter and in its first intron region, and RelA upregulates BECN1 transcriptional activity (37). We there- 
fore cotransfected a pGL3-BECN1 reporter vector, including $1.1 \mathrm{kbp}$ upstream of the human BECN1 promoter (37), together with RelA or RelB expression vectors into $293 \mathrm{~T}$ cells and performed dual luciferase assays. We confirmed RelA and RelB overexpression in 293T cells by WB (Figure 8C). BECN1 transcriptional activity was upregulated significantly by RelB, but only minimally by RelA, overexpression (Figure 8C), which may reflect RelA protein levels being relatively low in our assays compared with published levels (37). RANKL markedly increased BECN1 transcriptional activity, consistent with the increased BECN1 protein level induced by RANKL (Figure 8C). However, RANKL did not increase BECN1 activity further when RelA or RelB was overexpressed. Furthermore, siRNA knockdown of RelA or RelB in 293T cells resulted in reduced protein levels and an approximately 50\% reduction in BECN1 promoter activity (Figure 8D). To further assess interaction of RelB with the BECN1 promoter, we performed ChIP assays. An A $\kappa B$ site is conserved in humans and mice (37), and real-time PCR and standard PCR analyses of ChIP DNA revealed that RelB binds to this $A \kappa B$ site with a lower binding ability than RelA. Neither nonspecific IgG nor primers covering the proximal BECN1 promoter region displayed detectable binding (Figure 8E).

\section{Discussion}

TRAF3, a relatively understudied TRAF family member, has been shown recently to act as an important multifunctional regulator of type I IFN and balanced cytokine production by serving as an adaptor molecule that facilitates assembly of a NIK/TRAF2/ activated/cellular inhibitor of apoptosis (cIAP) complex (38). NIK plays a key role in noncanonical NF- $\mathrm{B}$ signaling, and its stability is regulated by TRAF3 in B cells (17) and in OCPs (11). Here, we have shown using gain- and loss-of-function approaches that TRAF3 suppresses RANKL-induced OC formation, consistent with TRAF3 being a negative regulator of noncanonical signaling (11). Mice that we generated with conditional KO of TRAF3 in OCPs were osteoporotic with increased OC numbers (Figure 1, $\mathrm{D}$ and $\mathrm{E}$ ), indicating that TRAF3 plays an important protective role in physiological bone remodeling.

Our finding that TRAF3 also inhibits expression of p65 is consistent with the increased $\mathrm{p} 65$-binding activity in Traf3 $3^{-/-}$cells (39) and increased phospho-p65 level in TRAF3 knockdown cells in response to TNF or LT $\beta R$ (40), but the mechanism whereby TRAF3 inhibits NF-KB signaling is unclear. TRAF3 deficiency did not alter protein levels of RANK or DC-STAMP (Supplemental Figure 7, $A$ and $B$ ), key regulators of RANKL-induced OCP differentiation and fusion $(41,42)$. TRAF3 could inhibit both NF- $\kappa$ B pathways by affecting NIK-IKK $\alpha$ signaling because IKK $\alpha$ is required for formation of IKK $\alpha / \operatorname{IKK} \beta / \mathrm{IKK} \gamma$ and IKK $\alpha / \mathrm{IKK} \alpha$ complexes in the canonical and noncanonical pathways, respectively $(43,44)$. In addition, TRAF3 overexpression upregulated expression of type I IFN (Supplemental Figure 7C), which can act as a negative regulator of osteoclastogenesis (45). Thus, TRAF3 might also suppress OC formation through RANKL/IFN- $\alpha$ signaling crosstalk. The primary role of canonical signaling appears to be to mediate OCP survival (46). However, when we inhibited RANKL-induced OC formation with CQ to prevent TRAF3 lysosomal degradation, we found no change in OCP proliferation or apoptosis rates (Figure 3, $\mathrm{C}$ and D), despite seeing a CQ-induced reduction in levels of the p65 target gene NFATc1 (Figure 4A) and a reduction in p65 levels in OCPs when TRAF3 was overexpressed (Figure 1F). Further studies will be required to determine exactly how TRAF3 functions in these NF-кB pathways and also whether it might have positive regulatory roles, as reported in other cell types (12).

In normal eukaryotic cells, autophagosomes digest damaged organelles and misfolded and long-lived proteins (47) and ultimately fuse with lysosomes, leading to degradation of their contents. Human genome-wide association data suggest a link between autophagy genes and osteoporosis (48), and the OC ruffled border membrane has been described as an autophagosome that fuses with lysosomes to facilitate secretion of matrix-degrading enzymes, especially cathepsin K. Furthermore, autophagy proteins, including Atg5, Atg7, Atg4B, and LC3, are important for generating the ruffled border (49). However, a potential role for autophagy in OC formation has not been reported. Here, we demonstrate for what we believe is the first time that autophagy also plays a role in osteoclastogenesis by regulating degradation of TRAF3, which we show undergoes ubiquitination and sequential autophagic-lysosomal degradation in response to RANKL. Our data are supported by a report that proteasome inhibition only marginally reduced TRAF3 degradation upon LT $\beta$ R stimulation in 293 T cells (50). However, TRAF3 can undergo K48-linked polyubiquitination by Triad $3 \mathrm{~A}$ and subsequent proteasomal degradation following virus infection in A549 human epithelial cells (51). Thus, the fate of TRAF3 may vary in response to different stimuli and be cell context dependent. In B cells, CD40 or BAFF receptor activation reduced TRAF3 degradation in a TRAF2/cIAP1/2dependent manner, and CD40-induced TRAF3 degradation was inhibited by a small-molecule mimetic, which inhibits cIAPs (52). We found that knockdown of cIAP1/2 reduced RANKL-induced TRAF3 degradation and decreased OC formation (Supplemental Figure 8), but the precise function of cIAPs in RANKL-induced TRAF3 lysosomal degradation will require further study.

Our finding that RelB is required for RANKL-induced TRAF3 lysosomal degradation is important because it points to a nega-

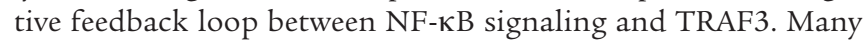
factors known to induce autophagy, including TNF (53), also act as NF- $\mathrm{B}$ activators. Depletion of essential autophagy modulators, including Atg5, Atg7, BECN1, and Vsp34 by RNAi inhibited TNFdriven NF-KB activation in human cancer cell lines (54). Similarly, $\mathrm{KO}$ of the genes encoding IKK $\alpha$, IKK $\beta$, IKK $\gamma$, or their upstream activator, TAK1, reduced autophagy induced by nutrient depletion or by treatment with rapamycin or other autophagy stimulators (55). However, the detailed molecular mechanisms are still unclear. We found that RANKL-induced TRAF3 degradation is absent in $\mathrm{RelB}^{-/-}$OCPs (Figure 8A). Our data suggest that RelB induction of TRAF3 lysosomal degradation is through transcriptional modulation of the early autophagic protein BECN1 (Figure 8E), with degradation of TRAF3 leading to activation of NF-KB signaling. Although the mechanism by which BECN1 might target TRAF3 for transfer to the lysosome is unclear, our findings have identified

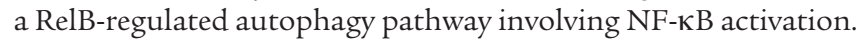

Given the essential functions of NF- $\mathrm{BB}$ in inflammation, autoimmune disease, and oncogenesis, large numbers of natural or synthetic NF-кB inhibitors have been produced (56). Although many of these inhibitors suppress osteoclastogenesis and bone resorption, none of them has progressed to clinical trials for the treatment of common bone diseases, such as postmenopausal osteoporosis or RA (4). Biologic inhibitors directed at pathogenetic cytokines, such as TNF, IL-1, and IL-6, have been approved for the treatment of inflammatory arthritis $(57,58)$. However, these treatments fail to achieve remission in up to $40 \%$ of patients (57). Thus, additional insights 
into the pathogenesis of joint destruction in this common disease and new drugs are needed. Our identification of TRAF3 as an inhibitor of osteoclastogenesis suggests a new target for treatment of RA.

CQ was used for many years to reduce inflammation in RA until it was replaced by hydroxychloroquine because the latter has fewer ophthalmic side effects. However, clinical studies indicate that, when given alone or in combination with methotrexate, hydroxychloroquine does not prevent joint destruction in $60 \%-70 \%$ of affected patients (58), suggesting that it may have limited use as an antiresorptive in most RA patients. CQ and hydroxychloroquine prevent acidification of the lysosomal compartment, but their mechanism of action is unclear, and reports of the effects of these drugs on OCs in vitro have been contradictory. For example, hydroxychloroquine had no inhibitory effect on human OCP differentiation (59), while CQ inhibited the formation of large OCs in the later phases of osteoclastogenesis (60). This latter finding differs from our in vitro observations and may reflect differences in culture conditions, but it does support a role for this class of compounds as inhibitors of bone resorption. Our finding that CQ inhibits OC formation in vitro and in vivo by preventing TRAF3 lysosomal degradation suggests that CQ could inhibit bone resorption in humans by this mechanism. The dose of CQ that we used in our in vivo studies $(50 \mathrm{mg} / \mathrm{kg})$ is higher than the dose of hydroxychloroquine given to patients $(\sim 5 \mathrm{mg} / \mathrm{kg})$, and this could account for the efficacy we observed in our mouse studies coupled with the fact that PTH- and OVX-induced resorption is less complex than the severe inflammatory milieu in RA. Recent studies indicate that CQ can have therapeutic efficacy in a number of other diseases, including cancer and pulmonary hypertension, and there are more than 30 clinical trials underway examining combinations of hydroxychloroquine with anticancer agents $(61,62)$. Other CQ derivatives could possibly have better anti-OC efficacy and fewer side effects, but further studies will be required to examine this.

In addition to this newly identified function of TRAF3 in bone homeostasis, TRAF3 has been implicated in the pathogenesis of a number of diseases, including multiple myeloma (63) and experimental autoimmune encephalomyelitis/multiple sclerosis (64). TRAF3 mutations in humans with multiple myeloma result in the accumulation of NIK and constitutive activation of NF- $\mathrm{KB}$, thereby promoting myeloma cell survival. Strategies to increase TRAF3 levels in OCPs by CQ or CQ derivatives should inhibit bone resorption in conditions in which RANKL is increased. Manipulating TRAF3 expression levels in other cell types, such as B cells, could also have beneficial therapeutic effects in patients with RA.

\section{Methods}

Mice. All animals were 6 to 12 weeks old. WT C57BL/6 (B6) mice were from the National Cancer Institute (Frederick, Maryland, USA). RelB-/- mice have been described previously (65). Traf3-floxed mice (B6 background) were from L. Rui (University of Michigan, Ann Arbor, Michigan, USA) (66). Lysozyme M-Cre (Lyz2tm1(cre)Ifo/J; B6 background) mice were purchased from the Jackson Laboratories. Cathepsin K-Cre mice (B6 background) were provided by Y.-P. Li (University of Alabama at Birmingham, Birmingham, Alabama, USA). To generate cKO mice, Traf3-floxed mice were bred with cathepsin K-Cre or lysozyme M-Cre transgenic mice. Cathepsin K-Cre, lysozyme M-Cre, and Traf3-floxed mice all have normal bone phenotypes (BV/ tissue volume [TV], Tb. N, Tb.Th, and Tb. Sp values; data not shown). Crenegative littermates were used as WT controls in all in vivo experiments.

$A b s$ and reagents. The following Abs were from Santa Cruz Biotechnology Inc.: TRAF3 (clone M20), NFATc1 (clone 7A6), c-Fos (clone 4),
NIK (clone H-248), RelB (clone C19), p65 (clone C20), p50 (clone NLS), and BECN1 (clone H-300). p100/p52 Ab was from Millipore. HA (clone $\mathrm{H} 6908$ ) and $\beta$-actin (clone AC-40) Abs were from Sigma-Aldrich. Recombinant M-CSF and RANKL were from R\&D Systems. MG132, bafilomycin A1, 3-MA, $\mathrm{NH}_{4} \mathrm{Cl}$, and CQ were from Sigma-Aldrich. LAMP2 Ab, clone GL2A7, was from Abcam.

Plasmid construction and retroviral transfer $A b s$ and reagents. The retroviral vector PMX-TRAF3-IRES-GFP WT and mutants were generated by inserting a cDNA fragment encoding TRAF3 WT and mutants into a PMX-IRES-GFP retroviral expression vector from K. Matsuo (Keio University, Minato, Tokyo, Japan). A PMX-TRAF3-GFP fusion protein vector was generated by inserting a TRAF3 PCR fragment into BamHI-NcoI sites of PMX-TRAF3-IRES-GFP. pMY-GFP vector, was from A. Hirao (Keio University). We generated PMY-RelB-GFP by inserting a RelB cDNA fragment into the pMY-GFP vector. MSCV-Cre and MSCV-Cre-GFP were from T. Reya (UCSD, La Jolla, California, USA). Retrovirus packaging was performed by transfecting plasmids into Plat-E cells (pMY virus) or $293 \mathrm{~T}$ cells (MSCV virus) using Fugene 6 (Roche) following the manufacturer's protocol. WT and mutant TRAF3 constructs were from S.C. Sun (17) (University of Texas, MD Anderson Cancer Center, Houston, Texas, USA). These plasmids were transfected into 293T cells using Fugene 6.

In vitro OC formation and functional assays. BM cells $\left(4 \times 10^{4}\right)$ were cultured in 96-well plates in $\alpha$-MEM with $10 \%$ FBS and recombinant M-CSF $(10 \mathrm{ng} / \mathrm{ml}$ in all experiments) for 2 days to enrich for OCPs. OCPs were infected with retrovirus for 2 days followed by RANKL $(10 \mathrm{ng} / \mathrm{ml}$ in all experiments, unless otherwise stated; R\&D Systems) for 5 to 7 days or treated with 2 to $10 \mu \mathrm{M}$ CQ and RANKL for 5 days. Osteoclastogenesis was assessed by counting $\mathrm{TRAP}^{+}$multinucleated cells with 3 or more nuclei. OC area was assessed using standard stereologic methods, an ocular eyepiece grid, an Olympus TH4-100 microscope, and a $\times 4$ objective lens. WT BM cells were cultured with M-CSF for 2 days on bone slices to generate OCPs and then CQ $(5 \mu \mathrm{M})$ and RANKL were added. Cells were fixed and stained for TRAP activity to allow counting of OCs, which were then removed; bone slices were stained with $0.5 \%$ toluidine blue to visualize resorption pits, as described (67).

In vitro CFU-F, CFU-ALP, and bone nodule formation assays. For CFU-F and CFU-ALP colony formation assays, BM cells from long bones were cultured in $10-\mathrm{cm}$ dishes with $10^{6} \mathrm{cells} / \mathrm{dish}$ in $10 \mathrm{ml} \alpha$-MEM plus $10 \% \mathrm{FCS}$ with or without $50 \mu \mathrm{g} / \mathrm{ml}$ ascorbic acid and $10 \mathrm{mM} \beta$-glycerophosphate. Media were changed every 3 to 4 days, and cultures were maintained for 24 days when cells were stained with H\&E or for ALP activity. For bone nodule formation, BM cells were cultured in $\alpha$-MEM plus 10\% FCS for 7 to 10 days and cultured in osteoblast-inducing medium containing $50 \mu \mathrm{g} / \mathrm{ml}$ ascorbic acid and $10 \mathrm{mM} \beta$-glycerophosphate for 21 to 28 days; mineralized bone nodules were examined after Von Kossa staining.

WB analysis. Whole-cell lysate protein from retrovirus-infected OCPs, TRAF3 plasmid-transfected 293T cells, WT, or cKO OCPs were cultured in 60-mm dishes and lysed with RIPA Lysis Buffer (Millipore) containing a protease inhibitor cocktail (Roche). Lysates $(10-40 \mu \mathrm{g})$ were loaded in $10 \%$ SDS-PAGE gels and immunoblotted with Abs to TRAF3, NFATc1, c-Fos, p65, RelB, p50, GAPDH, NIK, p100, HA, HDAC, BECN1, RANK, DC-STAMP, or mouse actin.

In vitro ubiquitination assays. WT BM cells were treated with M-CSF for 2 days and RANKL was added for 2 hours. Whole cell lysates were prepared using lysis buffer containing $20 \mathrm{mM}$ HEPES, $250 \mathrm{mM} \mathrm{NaCl}, 20 \mathrm{mM}$ Tris- $\mathrm{HCl}$, $0.5 \%$ NP-40, $2 \mathrm{mM}$ EDTA, $2 \mu \mathrm{g} / \mathrm{ml}$ leupeptin, $2 \mu \mathrm{g} / \mathrm{ml}$ aprotinin, $1 \mathrm{mM}$ DTT, $1 \mathrm{mM}$ PMSF, and $1 \mathrm{mM}$ N-ethylmaleimide (Sigma-Aldrich) to limit deubiquitination and incubated with UbiQapture-Q matrix (Enzo Life Sciences) to pull down all ubiquitinated proteins, following the manufacturer's protocol. Precipitates were subjected to WB using anti-TRAF3 Abs.

Flow cytometry and cell sorting. WT OCPs were infected with PMX-TRAF3GFP retrovirus for 3 days, and the cells were stained using an intracellular 
staining kit (BD Bioscience). In brief, the cells were fixed and permeabilized by Cytofix/Cytoperm buffer, then stained with PE-Traf $3 \mathrm{Ab}$ and Rat anti-mouse LAMP2 Ab (Santa Cruz Biotechnology Inc.). The secondary $\mathrm{Ab}$ (biotinylated anti-rat IgG; Vector Laboratories) was visualized by APC-streptavidin (BD Biosciences). WT OCPs were treated with RANKL and $10 \mu \mathrm{M} C Q$ for 3 days and stained with FITC-annexin V and propidium iodide (BD Biosciences) for 15 minutes. Cytometric analyses were performed using a flow cytometer (FACS LSR II; BD Biosciences) and FlowJo software. GFP-positive retrovirus-infected OCPs were sorted using FACSAria (BD Biosciences).

Immunocytochemistry and confocal microscopy. pMX-TRAF3-GFP-infected OCPs were seeded into 8-well chamber slides (Lab-Tek, NUNC), fixed with $4 \%$ paraformaldehyde, and permeabilized with $0.1 \%$ Triton X-100. After blocking with $5 \%$ goat serum in PBS for 4 hours, cells were incubated with anti-LAMP2 $\mathrm{Ab}(\mathrm{Abcam})$ in a humidity chamber at $4{ }^{\circ} \mathrm{C}$ overnight. The next day, cells were washed and incubated with secondary Alexa Fluor 350labeled anti-rat Ab. Colocalization of TRAF3 and LAMP2 in single cells was assessed using a FV1000 Olympus Laser Scanning Confocal Microscope.

In vivo PTH and CQ treatment and histological analysis. CQ $(50 \mathrm{mg} / \mathrm{kg} / \mathrm{d})$ was administered i.p. for 10 days to female WT or L-cKO mice (10 to 12 weeks old; 4/group), as described (68). On day 7 , mice were given supracalvarial injections of vehicle or hPTH(1-34 aa) (EMD Biosciences; $10 \mu \mathrm{g} /$ injection) $4 \times / \mathrm{d}$ for 3 days, (using a protocol that induces hypercalcemia and bone resorption; ref. 34), and killed 16 hours after the last injection. Calvarial and tibial sections were prepared as described (9). OC numbers and marrow fibrosis were measured in TRAP- and H\&E-stained sections using an eyepiece grid and an Olympus TH4-100 microscope.

Six-week-old female WT mice (7-8/group) were injected i.p. daily with $50 \mathrm{mg} / \mathrm{kg} \mathrm{CQ}$ and with hPTH ( $2 \mu \mathrm{g} /$ mouse s.c.) $3 \times / \mathrm{d}$ for 14 days to induce bone formation, as described (69). Tibiae were used for $\mu$ CT scanning. Calcein labeling was described previously (70). MAR and BFR in endocortical and trabecular bone were measured using standard procedures and Visiopharm Integrator System image analysis software.

OVX-induced bone loss. OVX or sham surgeries were performed in WT or C-cKO mice (8 to 9 weeks old; $5-9 /$ group). CQ treatment $(50 \mathrm{mg} / \mathrm{kg}$ i.p. daily injection for 28 days) was started 2 days after surgery. Controls received PBS. Calcein labeling was performed as described above. OVX was confirmed by uterine weight and histology. Tibiae were assessed by micro-CT and histomorphometry.

Electron microscopy. WT or RelB ${ }^{-/-}$OCPs were infected with Ctl-GFP, ATG5 shRNA, BECN1 shRNA, or ATG7 shRNA (Santa Cruz Biotechnology Inc.) for 2 days, cultured on glass chamber slides for 4 days with RANKL, fixed in $0.1 \mathrm{M}$ sodium cacodylate-buffered $2.5 \%$ glutaraldehyde for 24 hours, and post-fixed in $1 \%$ buffered osmium tetroxide for 30 minutes. Slides were transitioned through graded ethanols to $100 \%$ and infiltrated with Spurr's epoxy resin overnight. The slides were dipped into liquid nitrogen, and the entrapped cells were popped off and thin sectioned, then placed onto nickel grids, stained with aqueous uranyl acetate and lead citrate and examined using a Hitachi 7650 Transmission EM.

Reporter assay. A pGL3 luciferase reporter containing $1.1 \mathrm{kbp}$ of the human BECN1 promoter (CHET4) was from C. Schneider (Università degli Studi di Udine, Udine, Italy) (37). CHET4 and pcDNA3-RelA-cFlag (Addgene) or pcDNA3-RelB-cFlag plasmids (Addgene) was cotransfected into $293 \mathrm{~T}$ cells together with a Renilla luciferase plasmid with or without an hRANK construct. Cells were treated with RANKL or PBS, and dualluciferase assays were performed 24 hours after transfection. siRNA targeting RelA or RelB (Santa Cruz Biotechnology Inc.) was transfected into $293 \mathrm{~T}$ cells following the manufacturer's instructions. CHET4 and a Renilla luciferase plasmid were cotransfected by Fugene 648 hours later.

ChIP assay. ChIP was performed as described (37). In brief, BM-derived OCPs were treated with RANKL for 8 hours and fixed in $1 \%$ formaldehyde. Chromatin was sheared by sonication (16 rounds of 20 pulses with 2 minutes between rounds (Qsonica 125 sonicator). Then samples were incubated overnight at $4^{\circ} \mathrm{C}$ with Abs to p65, RelB, and IgG (negative control). Precipitated DNAs were analyzed by real-time PCR. Primers for the mIkBa promoter $(-316$ to -15$)$ were a positive control: $5^{\prime}$-GGACCCCAAAC-

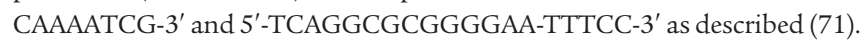
A primer set that covers the BECN1 proximal promoter region (-1897 to approximately -1513) was used as a negative control: 5'-GGGCAAGGCATCATAAAACAGG-3' and 5'-AGGAGATGAAGTTGACCTCC-3'. Specific primers for the $A \kappa B$ site in the BECN1 promoter region (-241 to approximately -1 ) are: $5^{\prime}$-AAGAAGCCTAGAGTCCCTGG- $3^{\prime}$ and $5^{\prime}$-CCTGCGACAGCGGAGAAAAG-3'.

Statistics. Values are shown as means + SEM of 4 samples unless otherwise stated. One-way ANOVA and nonparametric comparison (Bonferroni's multiple comparison test) were used with Prism software version 4.0b for experiments with more than 2 groups. Two-tailed Student's $t$ test was used for other statistical analyses, and significance was set at $P<0.05$.

Study approval. All animal experiments were performed using University of Rochester Medical Center ACUAC-approved protocols and conformed to the Guide for the Care and Use of Laboratory Animals (NIH. Revised 2011).

\section{Acknowledgments}

We thank Yanyun Li, Michael Thullen, and Karen Bentley for technical support. This work was supported by the following grants from the National Institute for Arthritis, Musculoskeletal and Skin Diseases: AR43510 and 1S10RR027340 (to B.F. Boyce); AR48697 (to L. Xing); P30AR061307, and T32AR053459.

Received for publication August 19, 2013, and accepted in revised form October 3, 2013.

Address correspondence to: Brendan F. Boyce, Department of Pathology and Laboratory Medicine, University of Rochester Medical Center, 601 Elmwood Avenue, Box 626, Rochester, New York 14642, USA. Phone: 585.275.5837; Fax: 585.273.3637; E-mail: Brendan_Boyce@URMC.Rochester.edu.

Yan Xiu's present address is: Department of Physical Medicine and Rehabilitation, Perelman School of Medicine, University of Pennsylvania, Philadelphia, Pennsylvania, USA.

Chen Zhao's present address is: Department of Pathology and Laboratory Medicine, University of Pennsylvania Medical Center, Philadelphia, Pennsylvania, USA

Yoshikazu Morita's present address is: Milk Science Research Institute, Megmilk Snow Brand Co., Saitama, Japan.
1. Sitara D, Aliprantis AO. Transcriptional regulation of bone and joint remodeling by NFAT. Immunol Rev. 2010;233(1):286-300.

2. Teitelbaum SL. Osteoclasts. What do they do and how do they do it? Am J Pathol. 2007;170(2):427-435.

3. Braun T, Zwerina J. Positive regulators of osteo- clastogenesis and bone resorption in rheumatoid arthritis. Arthritis Res Ther. 2011;13(4):235.

4. Boyce BF. Advances in osteoclast biology reveal potential new drug targets and new roles for osteoclasts. J Bone Miner Res. 2013;28(4):711-722.

5. Oeckinghaus A, Hayden MS, Ghosh S. Cross- talk in NF-KB signaling pathways. Nat Immunol. 2011;12(8):695-708.

6. Franzoso G, et al. Requirement for NF- $\kappa \mathrm{B}$ in osteoclast and B-cell development. Genes Dev. 1997; 11(24):3482-3496.

7. Iotsova $\mathrm{V}$, et al. Osteopetrosis in mice lacking NF-кB1 
and NF-кB2. Nat Med. 1997;3(11):1285-1289.

8. Vaira $S$, et al. RelB is the NF- $\kappa B$ subunit downstream of NIK responsible for osteoclast differentiation. Proc Natl Acad Sci U S A. 2008;105(10):3897-3902.

9. Yao Z, Xing L, Boyce BF. NF-кB p100 limits TNF-induced bone resorption in mice by a TRAF3-dependent mechanism. J Clin Invest. 2009; 119(10):3024-3034.

10. Zhao B, Grimes SN, Li S, Hu X, Ivashkiv LB. TNFinduced osteoclastogenesis and inflammatory bone resorption are inhibited by transcription factor RBP-J. J Exp Med. 2012;209(2):319-334.

11. Yang C, et al. NIK stabilization in osteoclasts results in osteoporosis and enhanced inflammatory osteolysis. PLoS One. 2010;5(11):e15383.

12. Hacker H, Tseng PH, Karin M. Expanding TRAF function. TRAF3 as a tri-faced immune regulator. Nat Rev Immunol. 2011;11(7):457-468.

13. Bishop GA. The multifaceted roles of TRAFs in the regulation of B-cell function. Nat Rev Immunol. 2004; 4(10):775-786

14. Rothe M, Sarma V, Dixit VM, Goeddel DV. TRAF2mediated activation of NF- $\kappa$ B by TNF receptor 2 and CD40. Science. 1995;269(5229):1424-1427.

15. Cao Z, Xiong J, Takeuchi M, Kurama T, Goeddel DV. TRAF6 is a signal transducer for interleukin-1. Nature. 1996;383(6599):443-446.

16. Xu Y, Cheng G, Baltimore D. Targeted disruption of TRAF3 leads to postnatal lethality and defective T-dependent immune responses. Immunity. 1996; 5(5):407-415.

17. Liao G, Zhang M, Harhaj EW, Sun SC. Regulation of the NF- $\mathrm{KB}$-inducing kinase by tumor necrosis factor receptor-associated factor 3-induced degradation. J Biol Chem. 2004;279(25):26243-26250.

18. He JQ, et al. Rescue of TRAF3-null mice by p100 NF-кB deficiency.JExp Med.2006;203(11):2413-2418.

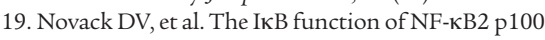
controls stimulated osteoclastogenesis. J Exp Med. 2003;198(5):771-781

20. Li P, et al. Systemic tumor necrosis factor alpha mediates an increase in peripheral CD11bhigh osteoclast precursors in tumor necrosis factor $\alpha$-transgenic mice. Arthritis Rheum. 2004;50(1):265-276.

21. Cheng $G$, et al. Involvement of CRAF1, a relative of TRAF, in CD40 signaling. Science. 1995; 267(5203):1494-1498.

22. He JQ, Saha SK, Kang JR, Zarnegar B, Cheng G. Specificity of TRAF3 in its negative regulation of the noncanonical NF-KB pathway. J Biol Chem. 2007; 282(6):3688-3694

23. Dadgostar $\mathrm{H}$, Cheng $\mathrm{G}$. An intact zinc ring finger is required for tumor necrosis factor receptor-associated factor-mediated nuclear factor- $\kappa \mathrm{B}$ activation but is dispensable for c-Jun $\mathrm{N}$-terminal kinase signaling. J Biol Chem. 1998;273(38):24775-24780.

24. Mizushima N. Autophagy: process and function. Genes Dev. 2007;21(22):2861-2873.

25. Vallabhapurapu $S$, et al. Nonredundant and complementary functions of TRAF2 and TRAF3 in a ubiquitination cascade that activates NIK-dependent alternative NF- $\mathrm{KB}$ signaling. Nat Immunol. 2008;9(12):1364-1370.

26. Clague MJ, Urbe S. Ubiquitin. same molecule, different degradation pathways. Cell. 2010; 143(5):682-685.

27. Ang E, et al. Proteasome inhibitors impair RANKLinduced NF-kappaB activity in osteoclast-like cells via disruption of $\mathrm{p} 62$, TRAF6, CYLD, and IкB $\alpha$ signaling cascades. J Cell Physiol. 2009;220(2):450-459.

28. Yamamoto A, et al. Bafilomycin A1 prevents maturation of autophagic vacuoles by inhibiting fusion between autophagosomes and lysosomes in rat hepatoma cell line, H-4-II-E cells. Cell Struct Funct. 1998;23(1):33-42.

29. Wu YT, et al. Dual role of 3-methyladenine in modulation of autophagy via different temporal patterns of inhibition on class I and III phosphoinositide 3-kinase. J Biol Chem. 2010; 285(14):10850-10861.

30. Ben-Zvi I, Kivity S, Langevitz P, Shoenfeld Y. Hydroxychloroquine. From malaria to autoimmunity. Clin Rev Allergy Immunol. 2012;42(2):145-153.

31. Tam CS, Heersche JN, Murray TM, Parsons JA. Parathyroid hormone stimulates the bone apposition rate independently of its resorptive action. differential effects of intermittent and continuous administration. Endocrinology. 1982;110(2):506-512.

32. Hock JM, Gera I. Effects of continuous and intermittent administration and inhibition of resorption on the anabolic response of bone to parathyroid hormone. J Bone Miner Res. 1992;7(1):65-72.

33. Ma YL, et al. Catabolic effects of continuous human PTH (1-38) in vivo is associated with sustained stimulation of RANKL and inhibition of osteoprotegerin and gene-associated bone formation. Endocrinology. 2001;142(9):4047-4054.

34. Yates AJ, et al. Effects of a synthetic peptide of a parathyroid hormone-related protein on calcium homeostasis, renal tubular calcium reabsorption, and bone metabolism in vivo and in vitro in rodents. J Clin Invest. 1988;81(3):932-938.

35. Klionsky DJ, et al. Guidelines for the use and interpretation of assays for monitoring autophagy in higher eukaryotes. Autophagy. 2008;4(2):151-175.

36. Levine B, Kroemer G. Autophagy in the pathogenesis of disease. Cell. 2008;132(1):27-42.

37. Copetti T, Bertoli C, Dalla E, Demarchi F, Schneider C. p65/RelA modulates BECN1 transcription and autophagy. Mol Cell Biol. 2009;29(10):2594-2608.

38. Tseng PH, et al. Different modes of ubiquitination of the adaptor TRAF3 selectively activate the expression of type I interferons and proinflammatory cytokines. Nat Immunol. 2010;11(1):70-75.

39. Zarnegar B, Yamazaki S, He JQ, Cheng G. Control of canonical NF- $\kappa B$ activation through the NIKIKK complex pathway. Proc Natl Acad Sci US A. 2008; 105(9):3503-3508.

40. Bista $P$, et al. TRAF3 controls activation of the canonical and alternative NFKB by the lymphotoxin beta receptor. J Biol Chem. 2010;285(17):12971-12978.

41. Simonet WS, et al. Osteoprotegerin. a novel secreted protein involved in the regulation of bone density. Cell. 1997;89(2):309-319.

42. Kukita T, et al. RANKL-induced DC-STAMP is essential for osteoclastogenesis. J Exp Med. 2004; 200(7):941-946.

43. Senftleben U, et al. Activation by IKK $\alpha$ of a second, evolutionary conserved, NF-KB signaling pathway. Science. 2001;293(5534):1495-1499.

44. Zandi E, Rothwarf DM, Delhase M, Hayakawa M, Karin M. The IкB kinase complex (IKK) contains two kinase subunits, IKK $\alpha$ and IKK $\beta$, necessary for

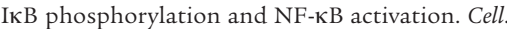
1997;91(2):243-252.

45. Takayanagi $\mathrm{H}$, et al. RANKL maintains bone homeostasis through c-Fos-dependent induction of interferon- $\beta$. Nature. 2002;416(6882):744-749.

46. Vaira S, et al. RelA/p65 promotes osteoclast differentiation by blocking a RANKL-induced apoptotic JNK pathway in mice. J Clin Invest. 2008; 118(6):2088-2097.

47. Deretic V, Jiang S, Dupont N. Autophagy intersections with conventional and unconventional secretion in tissue development, remodeling and inflammation. Trends Cell Biol. 2012;22(8):397-406.

48. Zhang L, et al. Pathway-based genome-wide association analysis identified the importance of regulation-of-autophagy pathway for ultradistal radius BMD. J Bone Miner Res. 2010;25(7):1572-1580.

49. DeSelm CJ, et al. Autophagy proteins regulate the secretory component of osteoclastic bone resorption. Dev Cell. 2011;21(5):966-974.

50. Ganeff C, et al. Induction of the alternative NF-кB pathway by lymphotoxin $\alpha \beta(\operatorname{LT} \alpha \beta)$ relies on internalization of LT $\beta$ receptor. Mol Cell Biol. 2011;
31(21):4319-4334.

51. Nakhaei P, et al. The E3 ubiquitin ligase Triad3A negatively regulates the RIG-I/MAVS signaling pathway by targeting TRAF3 for degradation. PLoS Pathog. 2009;5(11):e1000650

52. Zarnegar BJ, et al. Noncanonical NF- $\kappa$ B activation requires coordinated assembly of a regulatory complex of the adaptors cIAP1, cIAP2, TRAF2 and TRAF3 and the kinase NIK. Nat Immunol. 2008; 9(12):1371-1378

53. Djavaheri-Mergny $M$, et al. NF- $\kappa B$ activation represses tumor necrosis factor- $\alpha$-induced autophagy. J Biol Chem. 2006;281(41):30373-30382.

54. Criollo A, et al. Autophagy is required for the activation of NFKB. Cell Cycle. 2012;11(1):194-199.

55. Criollo A, et al. The IKK complex contributes to the induction of autophagy. EMBO J. 2010; 29(3):619-631.

56. Ben-Neriah Y, Karin M. Inflammation meets cancer, with NF-кB as the matchmaker. Nat Immunol. 2011; 12(8):715-723.

57. Smolen JS, Aletaha D, Koeller M, Weisman MH, Emery P. New therapies for treatment of rheumatoid arthritis. Lancet. 2007;370(9602):1861-1874.

58. Davila L, Ranganathan P. Pharmacogenetics. implications for therapy in rheumatic diseases. Nat Rev Rheumatol. 2011;7(9):537-550.

59. Lee CK, et al. Effects of disease-modifying antirheumatic drugs and antiinflammatory cytokines on human osteoclastogenesis through interaction with receptor activator of nuclear factor $\mathrm{\kappa B}$, osteoprotegerin, and receptor activator of nuclear factor кB ligand. Arthritis Rheum. 2004;50(12):3831-3843.

60. Voronov I, et al. The R740S mutation in the V-ATPase a3 subunit increases lysosomal $\mathrm{pH}$, impairs NFATc1 translocation and decreases in vitro osteoclastogenesis. J Bone Miner Res. 2013; 28(1):108-118.

61. Kimura T, Takabatake Y, Takahashi A, Isaka Y. Chloroquine in cancer therapy. a double-edged sword of autophagy. Cancer Res. 2013;73(1):3-7.

62. Lotze MT, Maranchie J, Appleman L. Inhibiting autophagy. a novel approach for the treatment of renal cell carcinoma. Cancer J. 2013;19(4):341-347.

63. Keats JJ, et al. Promiscuous mutations activate the noncanonical NF- $\mathrm{KB}$ pathway in multiple myeloma. Cancer Cell. 2007;12(2):131-144.

64. Xiao Y, et al. Peli1 promotes microglia-mediated CNS inflammation by regulating Traf3 degradation. Nat Med. 2013;19(5):595-602.

65. Burkly L, et al. Expression of relB is required for the development of thymic medulla and dendritic cells. Nature. 1995;373(6514):531-536

66. Gardam S, Sierro F, Basten A, Mackay F, Brink R. TRAF2 and TRAF3 signal adapters act cooperatively to control the maturation and survival signals delivered to B cells by the BAFF receptor. Immunity. 2008;28(3):391-401.

67. Zhang Q, et al. VEGF-C, a lymphatic growth factor, is a RANKL target gene in osteoclasts that enhances osteoclastic bone resorption through an autocrine mechanism. J Biol Chem. 2008; 283(19):13491-13499.

68. Parkhitko A, et al. Tumorigenesis in tuberous sclerosis complex is autophagy and p62/sequestosome 1 (SQSTM1)-dependent. Proc Natl Acad Sci U S A. 2011;108(30):12455-12460.

69. Calvi LM, et al. Osteoblastic expansion induced by parathyroid hormone receptor signaling in murine osteocytes is not sufficient to increase hematopoietic stem cells. Blood. 2012;119(11):2489-2499.

70 . Schwarz EM, et al. Tumor necrosis factor- $\alpha /$ nuclear transcription factor- $\mathrm{\kappa B}$ signaling in periprosthetic osteolysis. J Orthop Res. 2000;18(3):472-480.

71. Yamamoto Y, Verma UN, Prajapati S, Kwak YT, Gaynor RB. Histone H3 phosphorylation by IKK- $\alpha$ is critical for cytokine-induced gene expression. Nature. 2003;423(6940):655-659. 